\title{
From Solid to Plane Tessellations, and Back
}

\author{
Vera Viana ${ }^{1,2}$
}

\begin{abstract}
In solid tessellations or three-dimensional honeycombs, polyhedra fit together to fill space, so that every face of each polyhedron belongs to another polyhedron. Solid and plane tessellations are intrinsically connected, since any section cut through a solid tessellation always produces some kind of plane tessellation. To clarify this relation, we will mention a short list of convex polyhedra that fill space monohedrally and illustrate the convex uniform honeycombs, focusing on those with structural potential to outline spaceframes. With regular plane tessellations as starting point, we hint at the geometrical possibilities in which the Platonic and two Archimedean solids are explorable in topological interlocking, aiming to expand the repertoire of blocks for monohedral topological interlocking assemblies. This has possible applications in architecture, in relation, for example, to space frames.
\end{abstract}

Keywords Solid tessellations · Plane tessellations · Regular polyhedra · Quasiregular polyhedra · Topological interlocking

\section{Solid Tessellations}

The enumeration of polyhedra that fill space in infinite replicas (in other words, plesiohedra, whose centroids outline lattice points), Grünbaum and Shepard denote, "has no finite answer" (1980: 966) and remains an open problem in mathematics. In 1980, two types of asymmetrical convex polyhedra with thirty-eight faces, each of

\section{Vera Viana}

veraviana@veraviana.net

1 CEAU (Study Center of Architecture and Urbanism) - FAUP (Faculty of Architecture of Porto's University), Via Panorâmica, Porto 4150-564 Porto, Portugal

2 Sciences and Technology School, Trás-os-Montes e Alto Douro’s University, Vila Real, Portugal 
which fill space monohedrally, were discovered by Peter Engel (Grünbaum and Shepard 1980: 965). The search goes on, but here we will focus on primary parallelohedra, convex uniform tessellations and some topological interlocking assemblies.

The six primary parallelohedra illustrated in Figs. 1, 2, 3, 4, 5 and 6 are convex polyhedra with centrally symmetric faces that fill space by translation of their replicas. The crystallographer Evgraf Fedorov listed, in 1885, the cube (representative of all rhombohedra), the semiregular hexagonal prism, the rhombic dodecahedron, the elongated dodecahedron and the truncated octahedron (Grünbaum 2010: 4) as all the possible combinatorial types of convex polyhedra that fill space in monohedral tessellations, without changing orientation. To these, in 1960 Stanko Bilinski added a second rhombic dodecahedron, additionally proving it as (the last) convex isozonohedron (Grünbaum 2010: 5). For every primary parallelohedron, there is a spatial set of segments with a common midpoint and the direction of its edges, known as the vector star (Coxeter 1973: 27), that categorizes primary parallelohedra as zonohedra. As well as every other zonohedron, parallelohedra can be extended or shortened, by changing the length of any vector of its star. The angles between vectors may also be changed, distorting the faces of polyhedra. In any case, its space-filling capabilities are preserved (Lalvani 1992) and this possibility, denotes Kappraff, "gives zonohedra an advantage over geodesic domes as building structures ... since it enables polyhedral structures to be built which fit form and function" (1990: 375-376). As an example, we recall polar zonohedra (Towle 1996) and the dome in Bruno Taut's Glass Pavilion built in 1914.

Solid and plane tessellations are intrinsically connected, since any section cut through the former "always produces a tessellation of some kind" (Pugh 1976: 48). Furthermore, most of the solid tessellations ${ }^{1}$ in Figs. 1, 2, 3, 4, 5 and 6 have, implicit, a regular plane tessellation (illustrated in continuous lines). Whether regular, quasiregular ${ }^{2}$ or irregular, every plane tessellation shown results from sectioning each honeycomb outlined by the representatives of the six convex parallelohedra with planes passing through some of its vertices. In dashed lines, examples of other producible non-regular tessellations are shown.

\footnotetext{
${ }^{1}$ The notations used in this paper are found in Grünbaum and Shepard (1987) and Grünbaum (1994). Notations for uniform plane tessellations describe the configuration of the regular polygons in each vertex. (3.3.3.3.3.3) or $\left(3^{6}\right)$, for instance, identifies the tessellation of six equilateral triangles around each vertex. Notations for uniform polyhedra describe the configuration and number of faces around each vertex. (3.3.3), (4.4.4), and (3.4.3.4), for instance, respectively identify the regular tetrahedron, the cube and the quasiregular cuboctahedron. In notations for uniform solid tessellations, the exponent denotes the number of polyhedra of each kind in each vertex. $(4.4 .4)^{8}$, for instance, identifies the tessellation of eight cubes around each vertex.

${ }^{2}$ Plane tessellations or two-dimensional honeycombs are "infinite sets of polygons fitting together to cover the whole plane just once, so that every side of each polygon belongs also to one other polygon" (Coxeter 1973: 58). In uniform tessellations, convex regular polygons intersect in isogonal vertices, described by Grünbaum and Shepard as "vertices of a single type" (1987: 59). In regular tessellations, all polygons are equal, while in quasiregular, two different polygons intersect in every edge. There are three regular tessellations: $\left(3^{6}\right),\left(4^{4}\right)$ and $\left(6^{3}\right)$, and one quasiregular tessellation: (3.6.3.6).
} 
Fig. 1 Sectioning stacked cubes perpendicularly to any edge produces the regular $\left(4^{4}\right)$. Sectioning perpendicularly to a space diagonal produces the regular $\left(3^{6}\right)$ and the quasiregular (3.6.3.6), if the plane contains the edges' midpoints

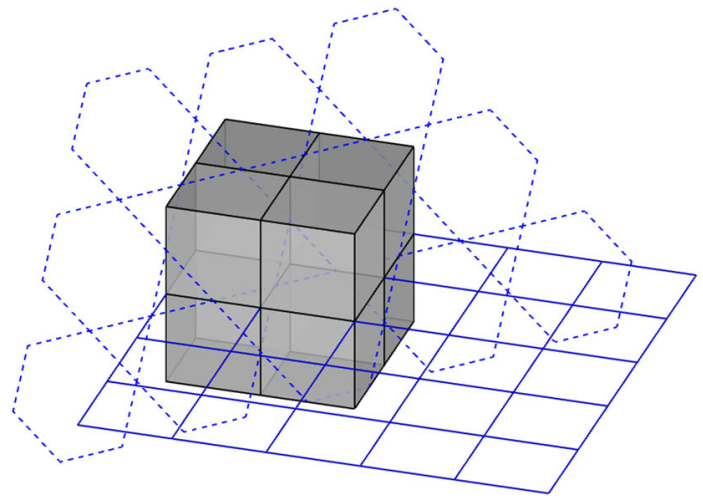

Fig. 2 Sectioning stacked hexagonal prisms perpendicularly to the sixfold symmetry axis produces the regular $\left(6^{3}\right)$

Fig. 3 Sectioning stacks of rhombic dodecahedra produces the regular $\left(4^{4}\right)$, if the plane is perpendicular to a fourfold symmetry axis
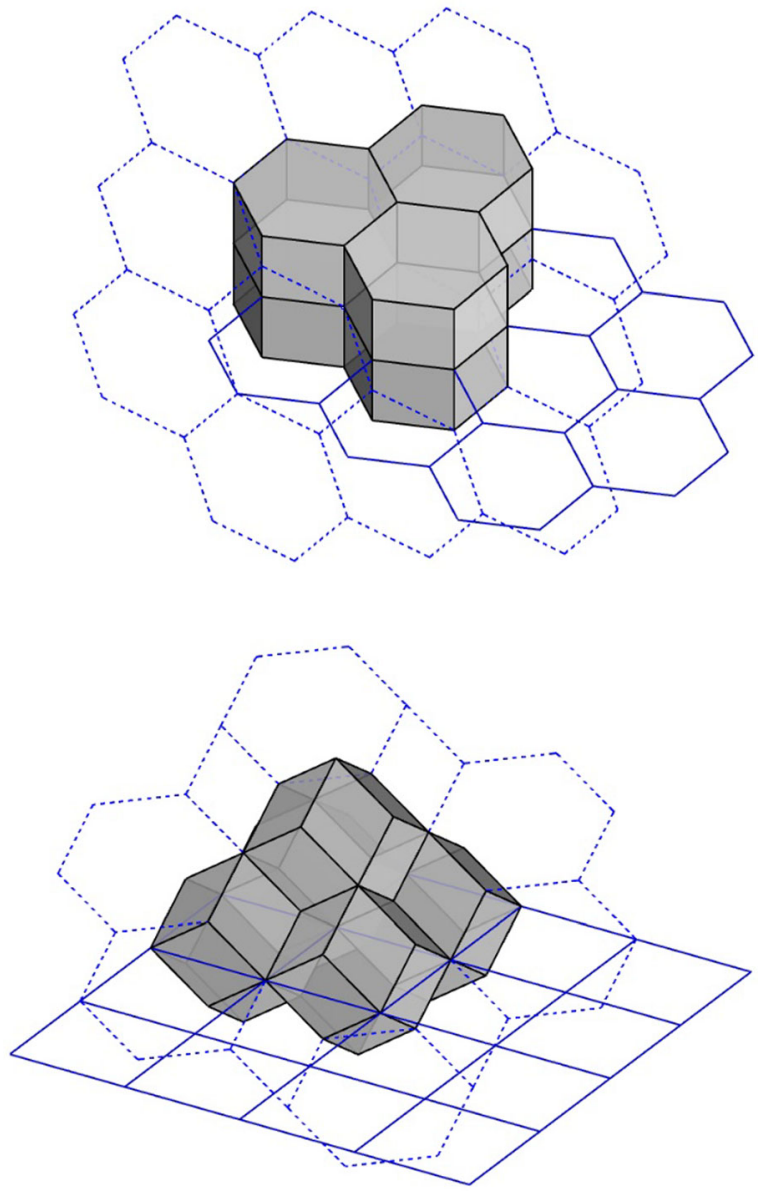
Fig. 4 Sectioning stacks of elongated dodecahedra produces the regular $\left(4^{4}\right)$, if the plane is perpendicular to a fourfold symmetry axis

Fig. 5 Sectioning stacks of truncated octahedra produces the regular $\left(4^{4}\right)$, if the plane is perpendicular to a fourfold symmetry axis and contains a long diagonal of the hexagonal faces

Fig. 6 There are no regularfaced tessellations producible by sectioning stacks of Bilinski's rhombic dodecahedra. The tessellation of irregular hexagons contains midpoints of some edges
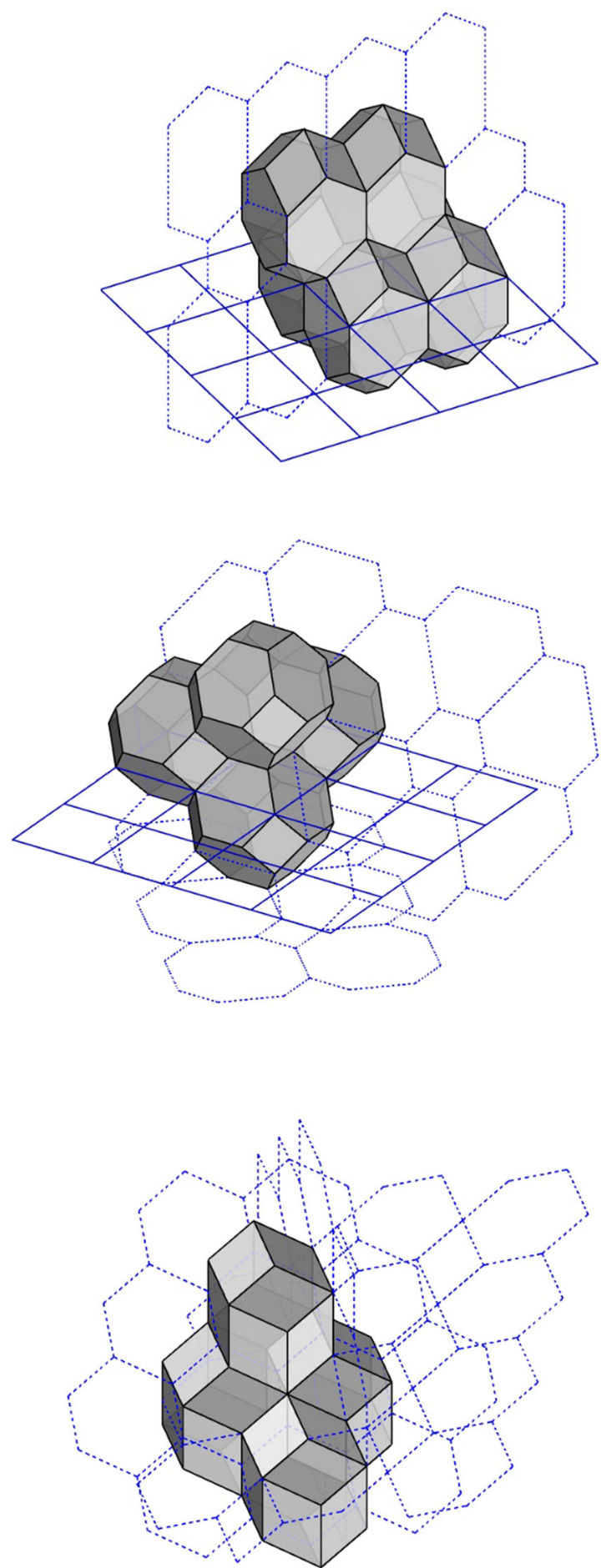


\section{Uniform Solid Tessellations}

In uniform solid tessellations, all the cells are convex uniform polyhedra and all vertices are transitive, that is, equally surrounded and forming "one orbit under symmetries" (Grünbaum 1994: 50). From convex uniform polyhedra, cubes, truncated octahedra and hexagonal prisms are space-fillers, as we have seen. Triangular semiregular prisms are capable of filling space as well, but they have to change orientation to do so. ${ }^{3}$ Given that distinct polyhedra must "have matching parallel faces in common" (Pearce 1978: 43), we may conclude that, to uniformly fill space with convex polyhedra, only cubes, tetrahedra, octahedra, cuboctahedra, truncated tetrahedra, truncated octahedra, truncated cubes, rhombicuboctahedra, rhombitruncated cuboctahedra and triangular, hexagonal, octagonal and dodecagonal prisms can perform efficiently as cells. In 1905, Alfredo Andreini published "On the regular and semiregular nets of polyhedra and on the corresponding correlative nets" with a systematic study of the 25 possibilities to close-pack polyhedra in uniform tessellations that he considered complete (although missing four and listing one mistakenly as uniform, according to Grünbaum (1994: 49)). Figure 7 illustrates one of his beautiful stereoscopic photographs. Keith Critchlow (1969) and Robert Williams (1979) published books in which this matter was illustrated but failed ${ }^{4}$ to consider the whole set of Andreini's tessellations. Peter Jon Pearce illustrated the same subject in 1978 but mentioned only twenty-three tessellations. ${ }^{5}$ In 1991, Norman Johnson extended the list to twenty-eight tessellations, an enumeration later confirmed as complete by Branko Grünbaum (1994), although this has not yet been proven so (Olshevsky 2006: 5). Until the end of the twentieth century, "mathematical literature was abundant with incomplete lists" of uniform partitions on three-dimensional space, according to Deza and Shtogrin (2000: 1). The intention of the following section and the "Appendix" at the end is to present the complete list of uniform tessellations and, whenever possible, its underlying uniform plane tessellations in order to broaden, if possible, the repertoire of geometrical possibilities for further researches in architecture, arts, design and engineering.

Grünbaum defines a slab as "the part of space between a pair of parallel planes" (1994: 52) and mentions that eighteen uniform tessellations simply consist of stacks of slabs. Of these, eleven are obtained from simple extrusion along the normal vector of each of the uniform plane tessellations (\#01, \#14, \#15, \#16, \#17, \#18, \#19, $\# 20$, \#21, \#22, \#23 in the "Appendix") and subsequent translation; two consist of

\footnotetext{
${ }^{3}$ Gyrobifastigia (Johnson's Solid J26) fill space, but they have to change orientation to do so (see \#27). However, they are not considered in the "Appendix", because each gyrobifastigium is, in fact, two joined semiregular triangular prisms.

${ }^{4}$ Critchlow (1969: Appendix 2) illustrates, besides seven non-uniform tessellations, twenty-one uniform tessellations corresponding, in our Appendix to: \#01, \#02, \#03, \#04, \#05, \#06, \#07, \#08, \#09, \#11, \#12, $\# 13$,\#14, \#15, \#16, \#17, \#18, \#19, \#20, \#22 and \# 23. Tessellation \#21 is illustrated, but with an incorrect diagram. Williams (1979: 167-182) illustrates, besides five non-uniform tessellations, fourteen uniform tessellations that correspond, in our Appendix, to: \#01, \#02, \#03, \#04, \#05, \#06, \#07, \#08, \#09, \#10, \#11, $\# 12$, \#13 and \#15. Tessellations \#14 to \#23 are mentioned (\#15 illustrated) and \# 21 is illustrated, with an incorrect diagram.

${ }^{5}$ Pearce (1978: 43-47) lists twenty-three uniform tessellations (eleven of them portrayed in beautiful photographs) but fails to mention the last five in our "Appendix".
} 


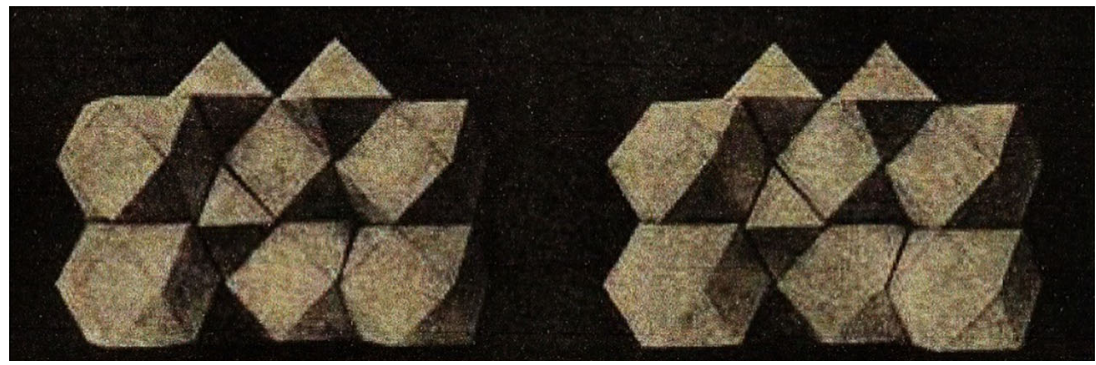

Fig. 7 Stereographic photograph of the tessellation outlined by cuboctahedra and octahedra. Image: (Andreini 1905, Fig. 18, pg. 108)

elongated or gyrated versions of the stacks of triangular prisms in \#14 (\#27, \#28); four involve octahedra in such positions that they are best regarded as triangular antiprisms (\#02, \#24, \#25, \#26); and the last one involves tetrahedra and truncated tetrahedra (\#03). The remaining ten tessellations are, as we shall see, a bit more elaborate.

\section{Architectonic and Catoptric Tessellations}

In this section, we briefly analyse the thirteen tessellations (\#01-\#13 in the "Appendix") that are considered analogues to uniform plane tessellations and categorised in Conway et al. (2008) as "architectonic tessellations", because "Architectonics is the theory of structural design and because its beginning reminds us of Archimedes" (2008: 292). The vertices of the dual cells for each tessellation, illustrated in Figs. 13, 14, 15, 16, 17, 18, 19, 20, 21, 22, 23, 24 and 25, are the centroids of all the polyhedra meeting face-to-face around each vertex. Through repeated reflection in every face, these cells outline the tessellation reciprocal to the first and fill space monohedrally. Furthermore, as illustrated in Conway et al. (2008: 294-298), each cell is a subdivision of the cube and/or the rhombic dodecahedron. The authors name these cells "catoptric chambers" and the duals of the architectonic tessellations are named "catoptric tessellations", "since Catoptrics is the theory of mirror-reflections and because its beginning reminds us of Catalan" (2008: 293). Eugène Catalan, we recall, published the first systematic account on the duality of polyhedra in 1865 (Malkevitch 1988: 88) and the duals of the Archimedean solids, Catalan solids, are named after him.

Of the convex regular polyhedra, only cubes fill space by translation of their replicas, outlining a tessellation in which eight cubes are stacked around each vertex, given that the amplitude of their dihedral angles is submultiple of $360^{\circ}$. As a space frame, the vertices of this regular (and self-dual) tessellation describe a simple cubic lattice with six vectors per vertex. The underlying plane tessellations are the regular $\left(4^{4}\right)$ or $\left(3^{6}\right)$ that we have seen in Fig. 1.

Tetrahedra and octahedra (whose dihedral angles are $70^{\circ} 31^{\prime} 44^{\prime \prime}$ and $109^{\circ} 28^{\prime} 16^{\prime \prime}$, respectively) fill space in two different arrangements, because their dihedral angles 
combined, add up to $180^{\circ}$ - a detail that fulfils the "self-evident necessary condition" mentioned by Kappraff (1990: 278) for polyhedra to fill space: that the sum of the dihedral angles of all polyhedra sharing a common edge equals $360^{\circ}$. Both tessellations comprise eight regular tetrahedra and six regular octahedra around each vertex, but while the former is quasirregular, the latter is not even uniform (and therefore not classifiable as architectonic). The central sphere in Figs. 8 and 9 stands for any vertex of each of the tessellations outlined by eight tetrahedra and six octahedra (for each of which, only eight edges and one equatorial square are shown). We may notice that the cuboctahedron, whose network Buckminster Fuller describes as the "isotropic vector matrix" (Kappraff 1990: 370), and the triangular orthobicupola (Johnson Solid J27) are clearly distinguishable as the convex hull of each set of tetrahedra. If we consider a sectioning plane for any face of the uniform version (Fig. 8), the plane tessellation produced is the regular $\left(3^{6}\right)$. This tetrahedral-octahedral tessellation describes a 12-connected network or

Fig. 8 Eight regular tetrahedra and six regular octahedra alternatively arranged around each vertex, each face belonging to a tetrahedron and an octahedron

Fig. 9 Eight regular tetrahedra and six regular octahedra in reflected layers around each vertex (the sequence of adjacent polyhedra is not the same in every edge)
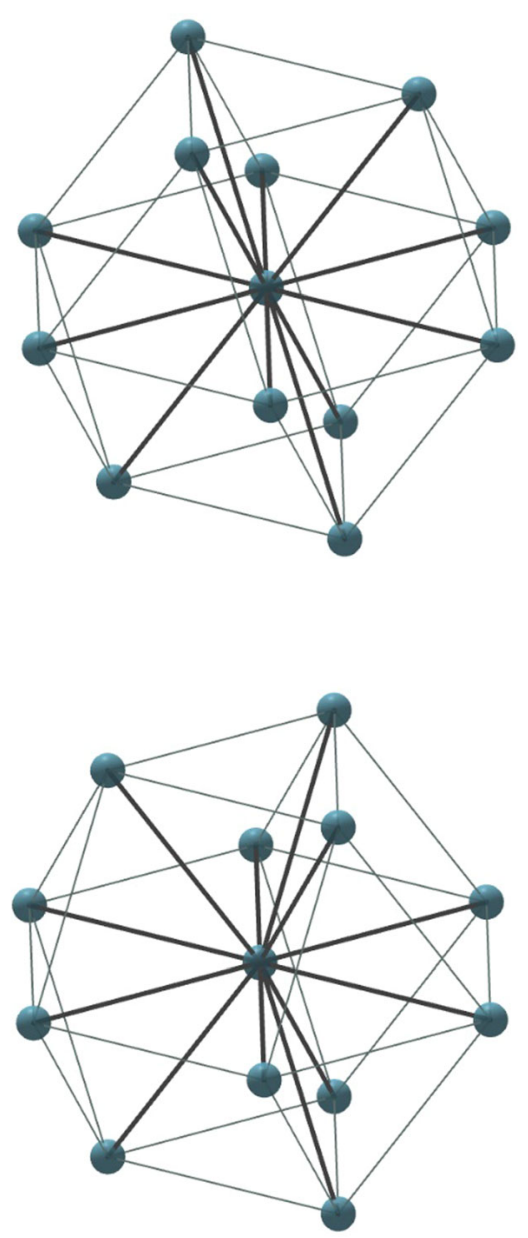
Fig. 10 The Voronoi cells of the simple cubic lattice are cubes

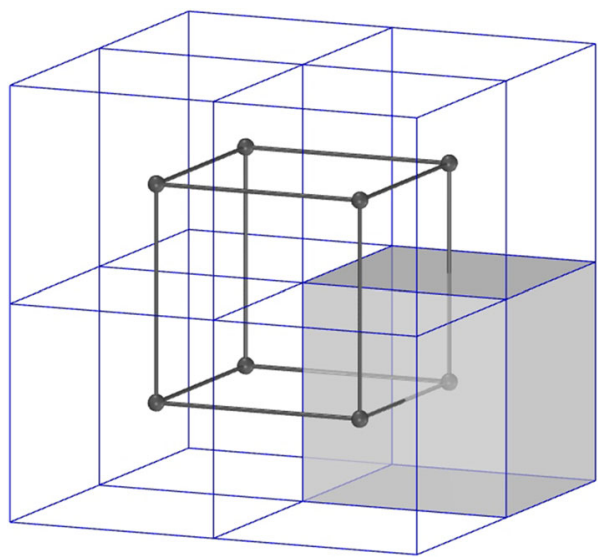

Fig. 11 The Voronoi cells of the face-centred cubic lattice are rhombic dodecahedra

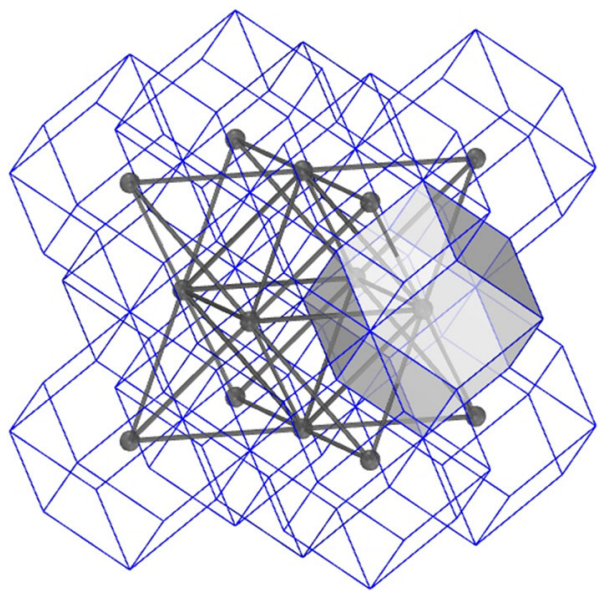

spaceframe that Gheorghiu and Dragomir describe as the "isotropic threedimensional grid of equidistant points along a maximum of directions symmetrically distributed in space" (1978: 231), patented in 1961 by Buckminster Fuller as the "octet truss". Although light in weight, it is a fully stable triangulated structure with high inherent rigidity and great efficiency in spanning volumes or planar regions, as demonstrated by Alexander Graham Bell, Buckminster Fuller, Robert le Ricolais, Louis Khan, Foster and Partners (Stansted Airport in London), Rod Robbie and Michael Allan (Rogers Centre in Ontario), John Allen, Phil Hawes and Peter Jon Pearce (Biosphere 2 in Arizona) as well as, among others, the Jacob K. Javits Convention Centre in New York and Sochi International Airport in Russia.

A small remark on point lattices, classified by Auguste Bravais in 1850 in the fourteen symmetry types connected to the seven types of coordinate systems or crystallographic unit cells (Chieh 1988: 99) to mention that the vertices of the tessellations we have just seen outline the three cubic lattices: the simple cubic, the 
Fig. 12 The Voronoi cells of the body-centred cubic lattice are truncated octahedra
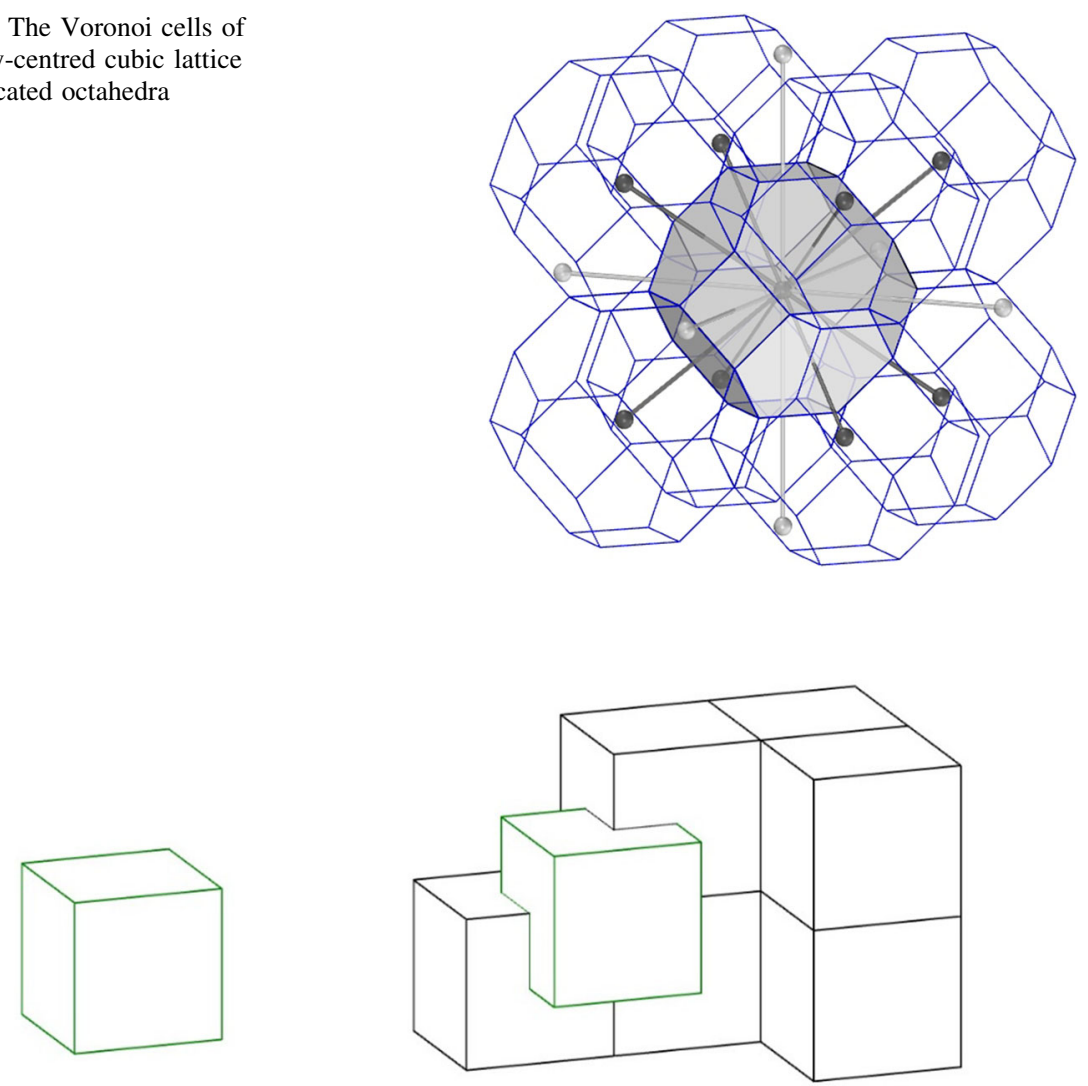

Fig. 13 The dual cells of the cubille tessellation (eight cubes per vertex) are cubes
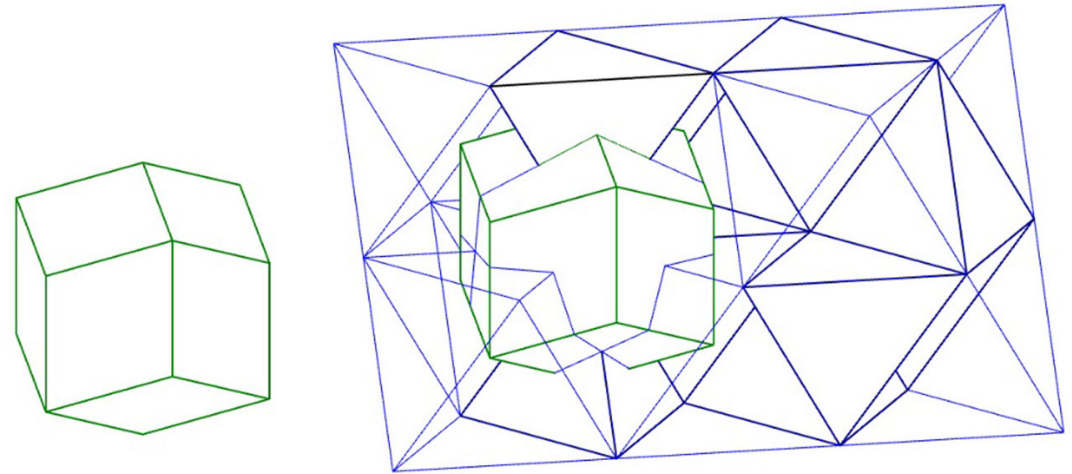

Fig. 14 The dual cells of the tetroctahedrille tessellation (eight tetrahedra and six octahedra per vertex) are rhombic dodecahedra 

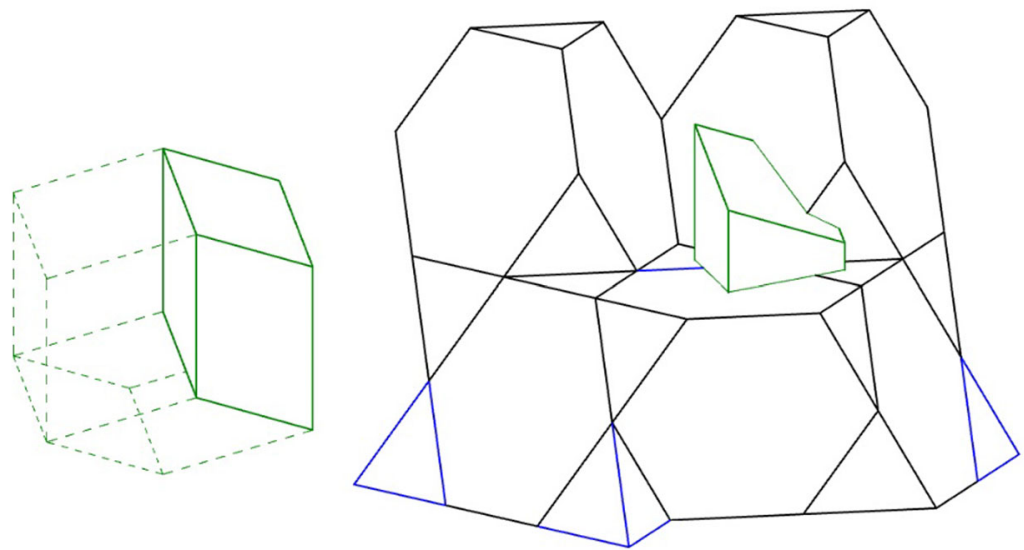

Fig. 15 The dual cells of the trunctetrahedrille tessellation (two tetrahedra and six truncated tetrahedra per vertex) are rhombohedra, four of which outline a rhombic dodecahedron
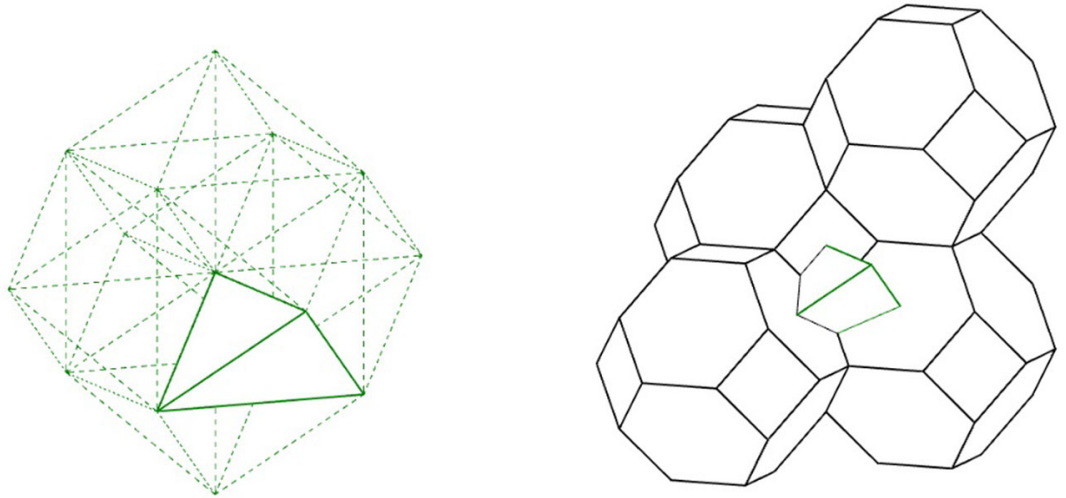

Fig. 16 The dual cells of the truncoctahedrille tessellation (four truncated octahedra per vertex) are tetragonal disphenoids, twenty-four of which outline a rhombic dodecahedron
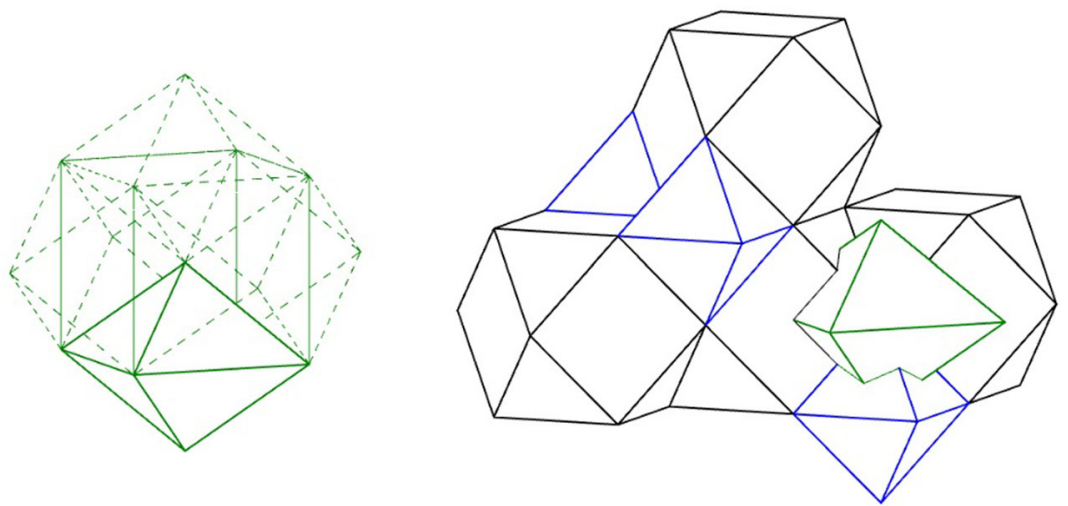

Fig. 17 The dual cells of the cuboctahedrille tessellation (two octahedra and four cuboctahedra per vertex) are square bipyramids with isosceles faces, six of which outline a rhombic dodecahedron 

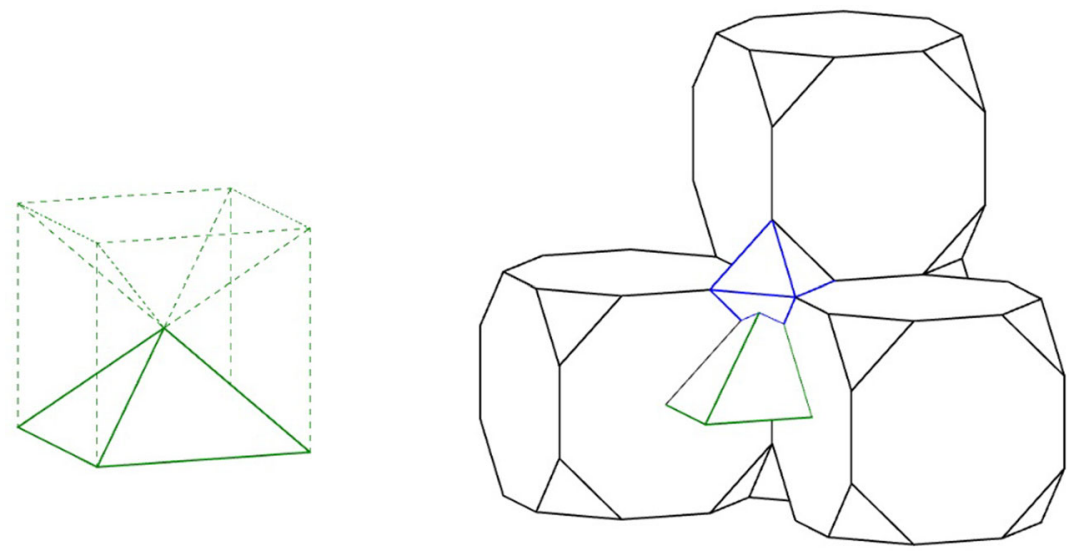

Fig. 18 The dual cells of the truncated cubille tessellation (one octahedron and four truncated cubes per vertex) are square pyramids with isosceles faces, six of which outline a cube
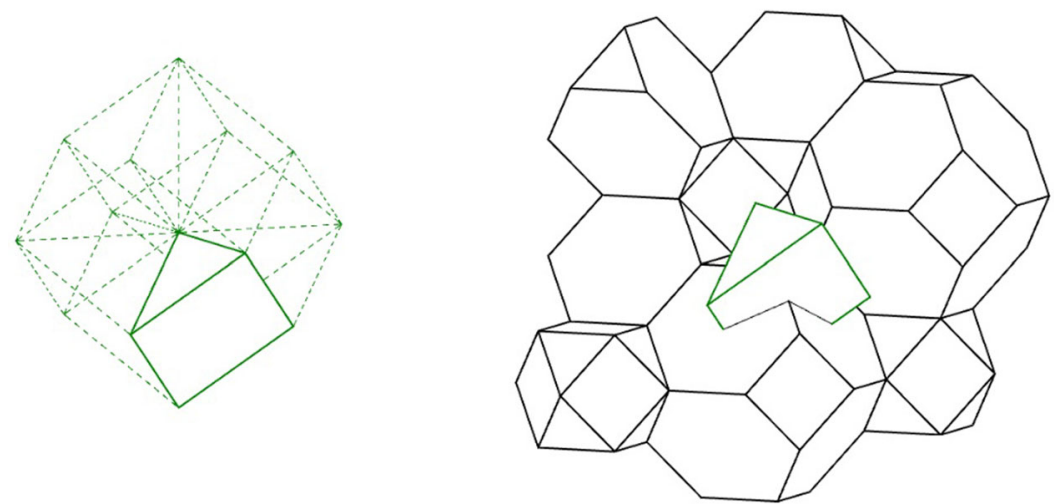

Fig. 19 The dual cells of the truncated tetroctahedrille tessellation (one cuboctahedron, two truncated tetrahedra and two truncated octahedra per vertex) are rhombic pyramids, twelve of which outline a rhombic dodecahedron

face-centred cubic and the body-centred cubic, respectively. The cells enclosed by the mediators of all the segments that connect any lattice point to its close neighbours (the Voronoi cell or Dirichlet region of each tessellation vertex) are cubes (Fig. 10) in simple cubic lattice and, in face-centred cubic and body-centred cubic lattices, rhombic dodecahedra (Fig. 11) and truncated octahedra (Fig. 12). The dihedral angles of the cube and the rhombic dodecahedron are respectively equal to $90^{\circ}$ and $120^{\circ}$, which explains why four and three of their replicas fill space around a vertex. Figure 12 intends to illustrate that the hexagonal faces of the truncated octahedron in the core belong to the mediator of the lines connecting the generator to its closest points (identified with the darker spheres), while the square faces belong to the mediating plane of the lines connecting the generator to the six outermost points (identified with the lighter spheres). In each edge, the sum of the dihedral angles between faces add up to $360^{\circ}$, since the angle between square and 

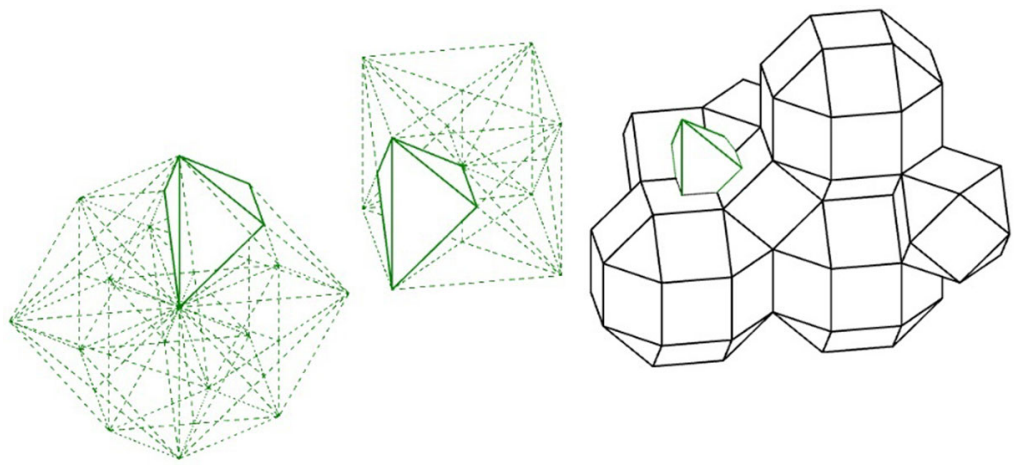

Fig. 20 The dual cells of the 2-RCO-hedrillle tessellation (one cuboctahedron, two rhombicuboctahedra and two cubes per vertex) are triangular bipyramids, twelve of each outline a cube, and twenty-four, a rhombic dodecahedron
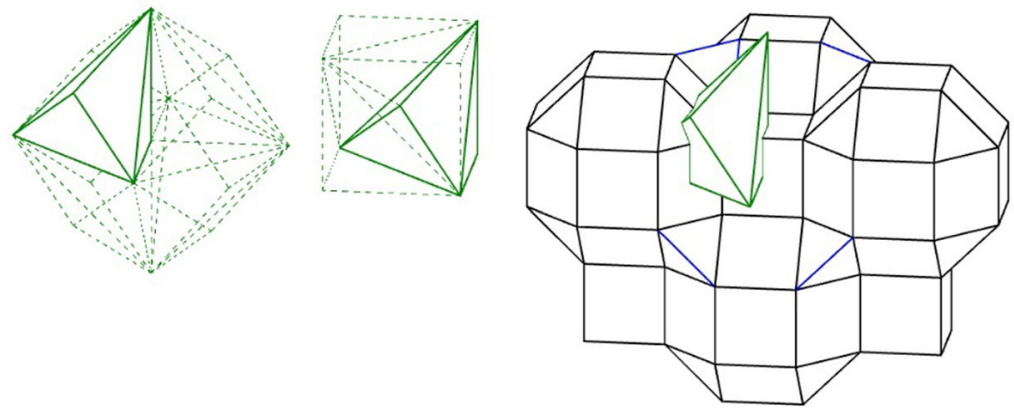

Fig. 21 The dual cells of the 3-RCO-hedrillle tessellation (one tetrahedron, three rhombicuboctahedra and one cube per vertex) are hexahedra, four of each outline a cube, and eight, a rhombic dodecahedron
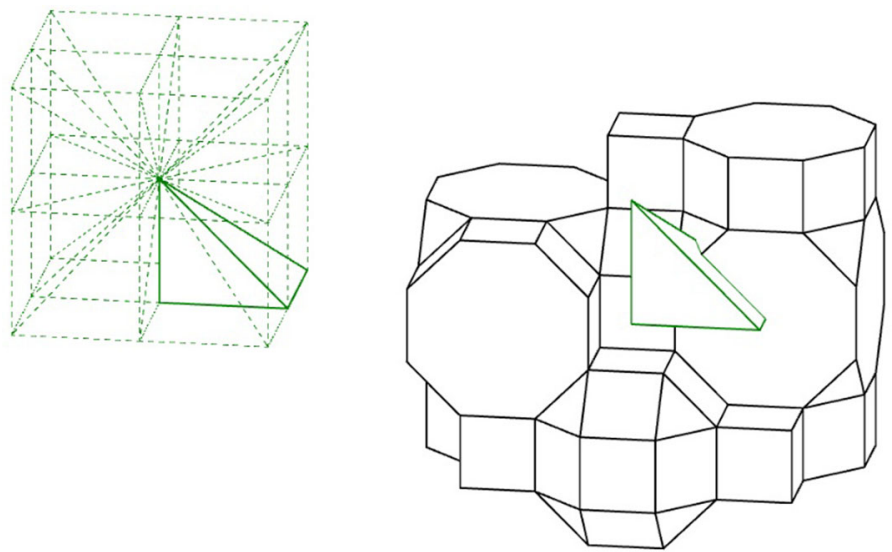

Fig. 22 The dual cells of the 1-RCO-hedrillle tessellation (one rhombicuboctahedron, one truncated cube, one cube and two octagonal prisms per vertex) are oblique square pyramids, twenty-four of each outline a cube 

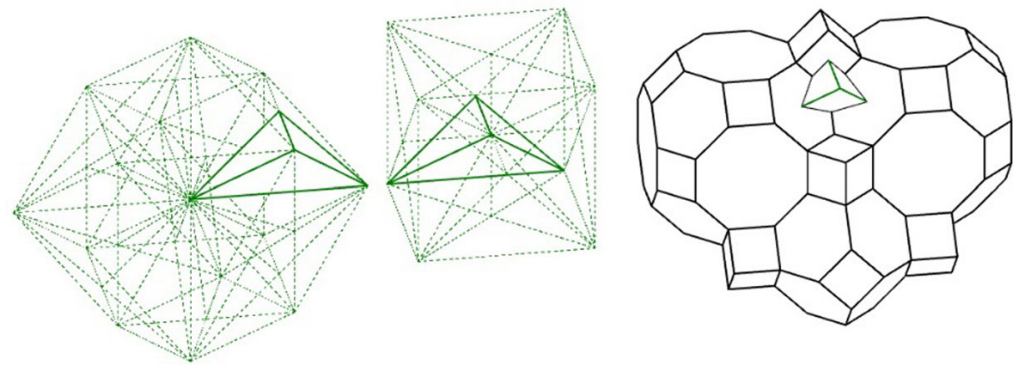

Fig. 23 The dual cells of the n-tCO-hedrille tessellation (one cube, one truncated octahedron and two rhombitruncated cuboctahedra per vertex) are triangular pyramids, twenty-four of each outline a cube, and forty-eight, a rhombic dodecahedron
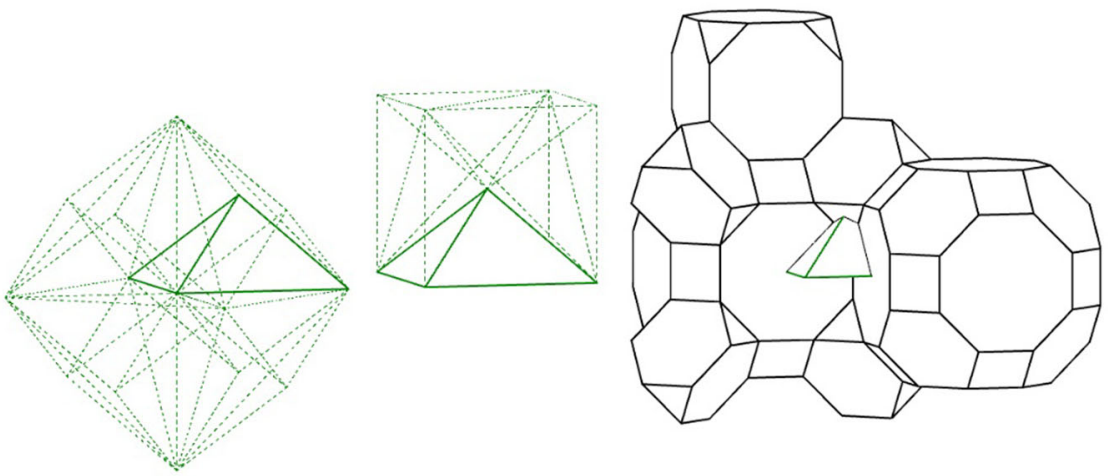

Fig. 24 The dual cells of the f-tCO-hedrille tessellation (one truncated tetrahedron, one truncated cube and two rhombitruncated cuboctahedra per vertex) are triangular pyramids, twelve of each outline a cube, and twenty-four, a rhombic dodecahedron
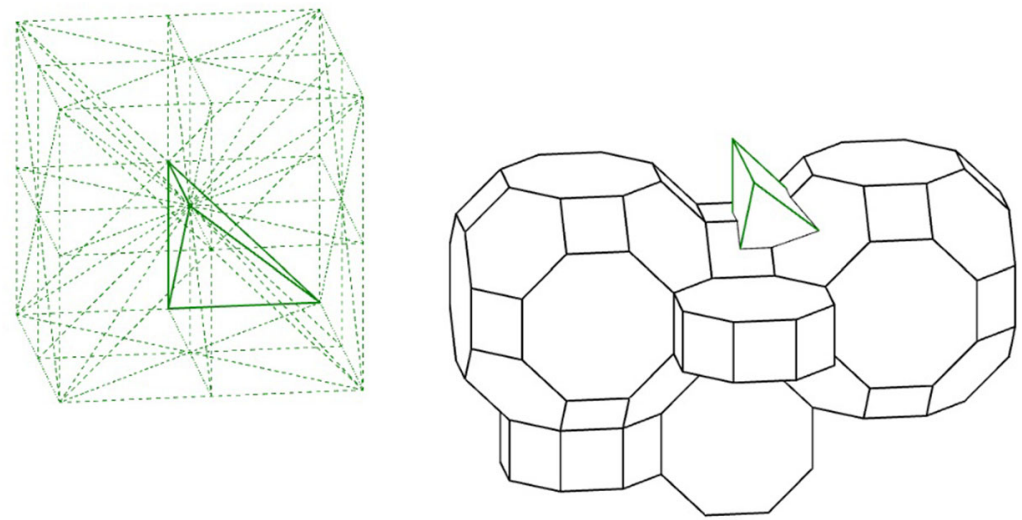

Fig. 25 The dual cells of the tessellation b-tCO-hedrille (two octagonal prisms and two rhombitruncated cuboctahedra per vertex) are triangular pyramids, forty-eight of which outline a cube 
hexagonal faces equals $125^{\circ} 15^{\prime} 52^{\prime \prime}$, and $109^{\circ} 28^{\prime} 16^{\prime \prime}$ between hexagonal faces. The truncated octahedra is unique, in the sense that "it gives the simplest decomposition of space in congruent parts" (Steinhaus 1960: 203) and the minimal partitional surface area for a given volume, thus extending "into three-dimensions the planar property of the regular hexagon" (Gheorghiu and Dragomir 1978: 231).

The remaining architectonic tessellations, which involve other uniform polyhedra besides the regular, are illustrated in Figs. 15, 16, 17, 18, 19, 20, 21, 22, 23, 24 and 25 , that present a fragment of each honeycomb and the corresponding dual or catoptric cell (whose edges are depicted in lighter lines). Given that all vertices are uniform, the dual cells are equal and, as such, the tessellation they outline is monohedral. The fact that their vertices coincide with the vertices of cubes and/or rhombic dodecahedra takes us once more to the simple cubic and face-centred cubic lattices. The designations of the tessellations in Figs. 13, 14, 15, 16, 17, 18, 19, 20, 21, 22, 23, 24 and 25 follow (Conway et al. 2008: 292-298).

\section{Regular Polygons from Convex Regular and Quasiregular Polyhedra}

If we arrange dual pairs of regular polyhedra so that their edges perpendicularly bisect each other, their common region is a quasiregular polyhedron. In other words, every quasiregular polyhedron is obtainable from the truncation at the midpoint's edges of any of the originating regular polyhedron. Here we will focus on the convex quasirregular: the Archimedeans cuboctahedron and icosidodecahedron, to which we add the Platonic octahedron, which, according to this definition, is also quasiregular, since its vertices are the midpoints of a dual pair of tetrahedra. In every edge of quasiregular polyhedra, two different regular polygons intersect, as each face is entirely surrounded by faces of a different kind. As such, all dihedral angles and edges' length are equal. Like every other uniform polyhedron, quasiregular polyhedra admit "symmetries which will transform a given vertex into every other vertex in turn" (Ball and Coxeter 1987: 136). But what differentiates them from the semiregular ${ }^{6}$ is not only the fact that all the edges are equally surrounded (unlike the former), but also that their equatorial polygons are inscribed in great circles of the corresponding circumsphere (Coxeter 1973: 18), which we believe is of great interest for this research. The equatorial polygons of the cuboctahedron are regular hexagons (confirming that the distance between the centroid and each vertex is the same as the edges' length ${ }^{7}$ ) and of the icosidodecahedron, regular decagons (the octahedron's equatorials are squares).

If we use vertices of a given polyhedron to outline new polygons, we are faceting the polyhedron. If these polygons (named facets) enclose a polyhedron, we are

\footnotetext{
${ }^{6}$ Semiregular polyhedra have regular faces and isogonal vertices. The convex set comprises: 2 infinite families of prisms and antiprisms, the truncated tetrahedron, the truncated cube, the truncated octahedron, the truncated dodecahedron, the truncated icosahedron, the rhombicuboctahedron, the rhombitruncated cuboctahedron, the rhombicosidodecahedron, the rhombitruncated icosidodecahedron, the snub cube and the snub dodecahedron, many of which were mentioned in the previous section. Designations follow (Wenninger 1971: 20-32).

7 The same occurs in the octahemioctahedron and cubohemioctahedron (quasiregular hemipolyhedra).
} 
obtaining a new polyhedron by faceting the first. To clarify the definition, we recall that two regular tetrahedra and its compound, the stella octangula, are obtained by faceting the cube and that the great stellated dodecahedron is obtained through faceting of the regular dodecahedron. The regular facets obtainable from convex regular polyhedra (with the obvious exception of the tetrahedron) are limited to ten-from the cube: triangles; from the octahedron: squares; from the dodecahedron: two kinds of triangles, squares, pentagons and pentagrams; from the icosahedron: triangles, pentagons and pentagrams (Cromwell 1997: 283). If we facet quasiregular polyhedra, we may obtain regular polygons as well-four from the cuboctahedron: triangles, squares, hexagons and hexagrams; and given its thirty vertices, twelve facets from the icosidodecahedron: two different kinds of triangles, squares, two different kinds of pentagons, three different kinds of pentagrams, hexagons, hexagrams, decagons and decagrams.

In addition to these regular polygons obtainable from four regular and two quasiregular polyhedra, we recall that, according to (Holden 1971: 23-27), the halfsection of the cube, the octahedron or the dodecahedron perpendicularly to any threefold axis of symmetry is a regular hexagon (a similar cross-section of the icosahedron is an equilateral decagon). A plane perpendicular to any fivefold symmetry axis, passing through the midpoints of the edges of the dodecahedron or the icosahedron, has a regular decagon for result. Sectioning the tetrahedron in half, perpendicularly to any of its twofold symmetry axis, has a square for result, as (Glickman 1984: 10) notes, although there it is mistakenly considered as an equatorial polygon. With edges' midpoints as vertices, there are no regular polygons obtainable from the cuboctahedron or the icosidodecahedron, because they have faces of two different kinds.

\section{Geometrical Possibilities for Convex Regular and Quasiregular Polyhedra to Topologically Interlock}

The purpose of this section is to expand the repertoire of possibilities for topological interlocked systems, "in which elements (blocks) of special shape are arranged in such a way that the whole structure can be held together by a global peripheral constraint" (Estrin et al. 2011: 1189). With this purpose in mind, we will analyse the geometrical possibilities for seven convex uniform polyhedra to perform as blocks of monohedral interlocked assemblies, considering the aforementioned regular facets and cross-sections as prototiles of regular plane tessellations. The reasons for choosing convex quasiregular polyhedra for topological interlocked structures (in addition to the regular set found in other researches) were not only the regularity of their (different) faces, but their edge-transitiveness, through which similar pairs of different faces intersect in every edge and the polyhedron looks exactly "the same when viewed edge on, from any edge" (Cromwell 1997: 371). In Figs. 26, 27, 28, $29,30,31,32,33,34,35,36,37,38,39,40,41,42,43,44,45$ and 46, whenever an interlocked assembly is not possible, only a few polyhedra are shown. The situations in which adjacent polyhedra share more than a face plane and an edge or vertex and a periodical interlocked assembly is feasible are illustrated with a layer of polyhedra resulting from the parallel translation of the initial set. 
Fig. 26 It is possible to assemble three cubes with triangular facets according to $\left(3^{6}\right)$, but coplanar faces have only one edge in common

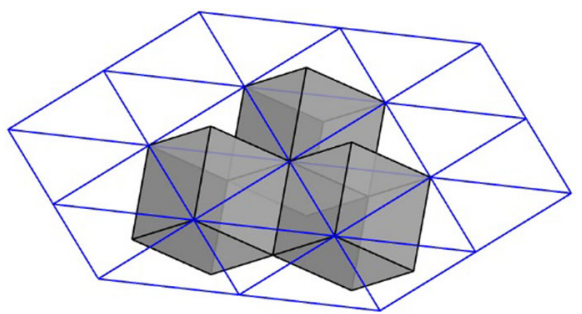

Fig. 27 It is not possible to assemble three dodecahedra with their smaller triangular facets according to $\left(3^{6}\right)$, without occurring intersections
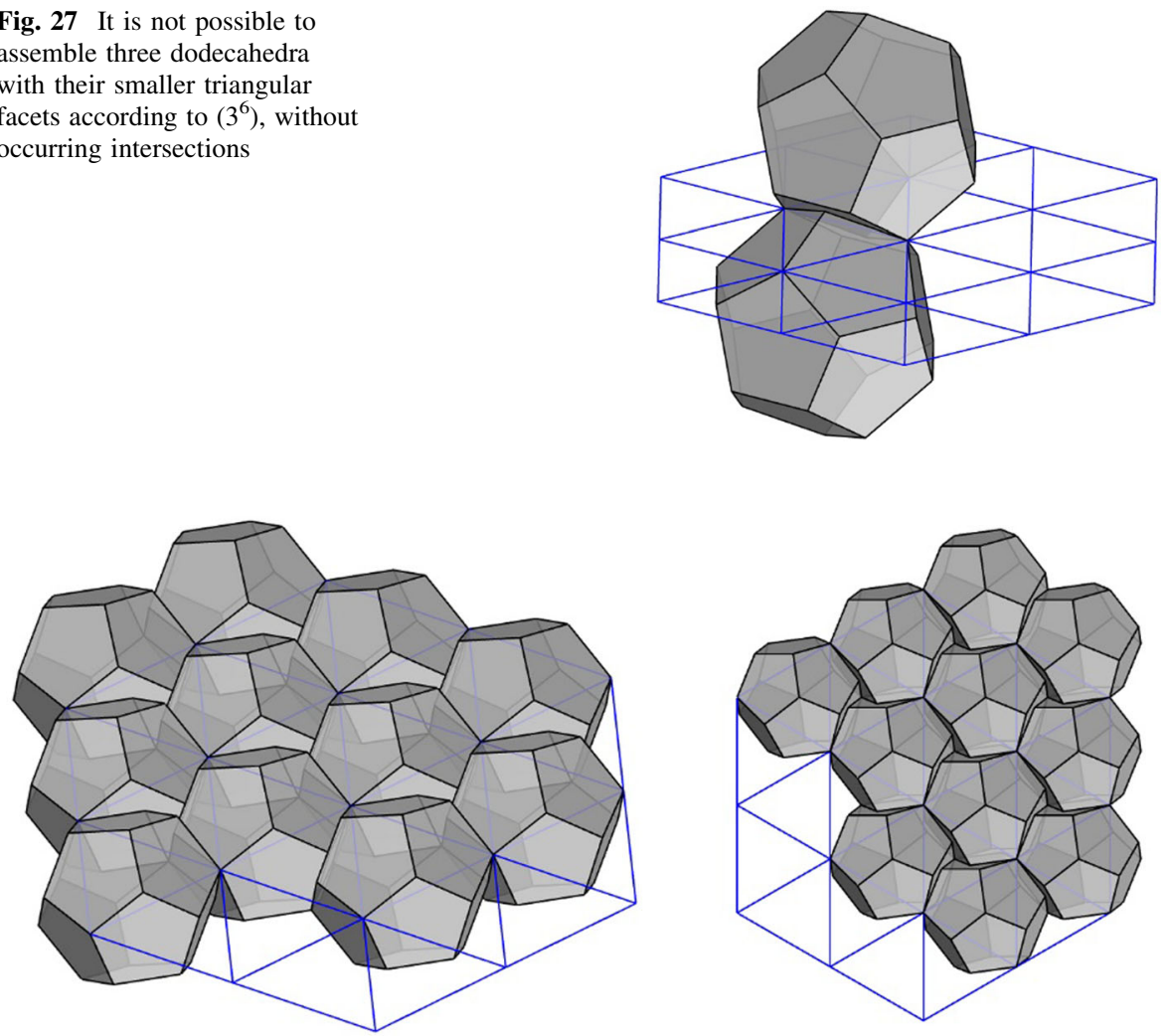

Fig. 28 Topological interlocking of dodecahedra with their larger triangular facets according to $\left(3^{6}\right)$ (perspective and top view)

For systematization purposes, the possibilities outlined from the regular tessellation $\left(3^{6}\right)$ are illustrated in Figs. 26, 27, 28, 29, 30, 31 and 32, but it should be mentioned that (Dyskin et al. 2003: 3) maintain that no arrangements thus obtainable should be considered as interlocking assemblies, since in every situation, no two pairs of faces of neighbouring blocks would exist to prevent each from moving upwards and downwards, meaning these possibilities cannot act as reference blocks of interlocking assemblies. The same authors clarify that an element of an assembly is locked by the geometrical constraints of its neighbours "if 
Fig. 29 Three icosahedra can be assembled with triangular facets according to $\left(3^{6}\right)$, but coplanar faces have only one edge in common

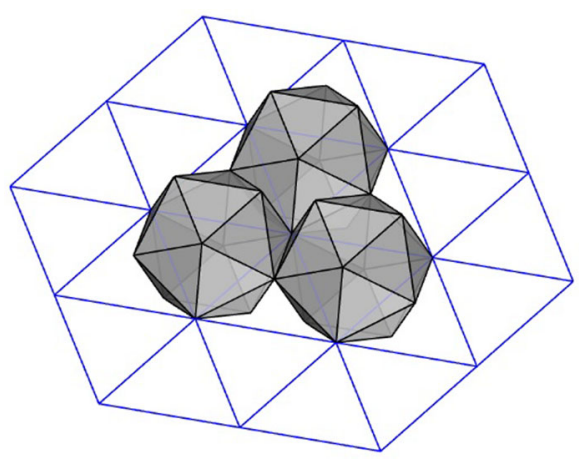

Fig. 30 It is possible to assemble three cuboctahedra with triangular facets according to $\left(3^{6}\right)$ (similarly to Fig. 41 ), but coplanar faces have only one edge in common

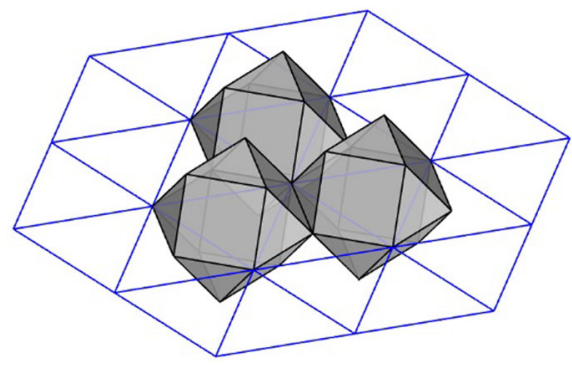

Fig. 31 It is not possible to assemble three icosidodecahedra with their smaller triangular facets according to $\left(3^{6}\right)$, without occurring intersections

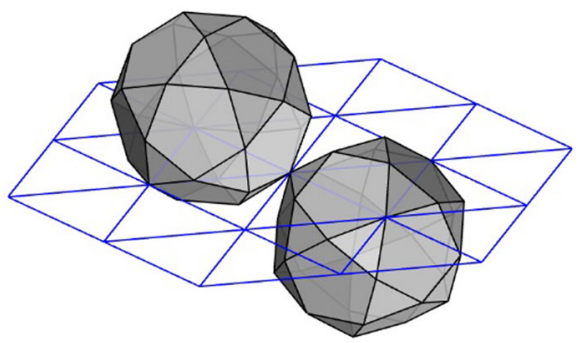

and only if, by continuously shifting the section plane in either direction, the corresponding polygon eventually degenerates to a segment or a point" (2003: 4). This procedure was later described as an "evolutionary transformation of the interlocking diagram" (Kanel-Belov et al. 2008: 10) and it was clarified that polygons in the section plane could evolve into a finite region, as, for instance, another polygon, as in Figs. 39 or 42 . Furthermore, no more than three polyhedra are able to interlock in each vertex of the tessellation $\left(3^{6}\right)$.

Even so, we consider Figs. 28 and 32 as interesting cases, since three dodecahedra or three icosidodecahedra with their larger triangular facets according to $\left(3^{6}\right)$ are able to interlock through planar translation without gaps nor intersections. Since the centroids of the dodecahedra in Fig. 28 describe a regular $\left(3^{6}\right)$ and all the sections perpendicular to the threefold symmetry axis degenerate into a vertex, the dodecahedra are topologically interlocked. This possibility 

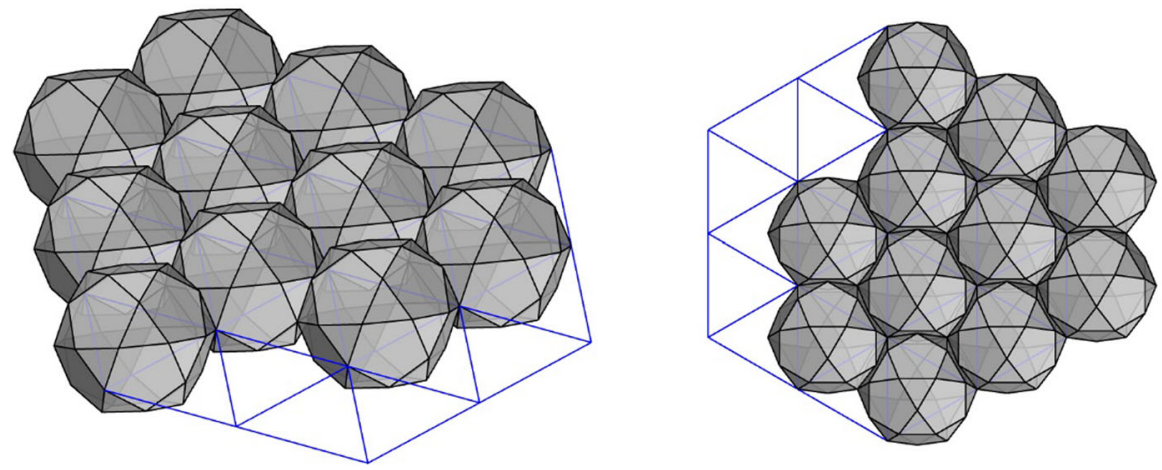

Fig. 32 Topological interlocking of icosidodecahedra with their larger triangular facets according to $\left(3^{6}\right)$ (perspective and top view)

Fig. 33 Topological

interlocking of tetrahedra with square cross-sections outlining $\left(4^{4}\right)$

Fig. 34 Octahedra can be assembled with square facets outlining $\left(4^{4}\right)$, but coplanar faces have only one edge in common
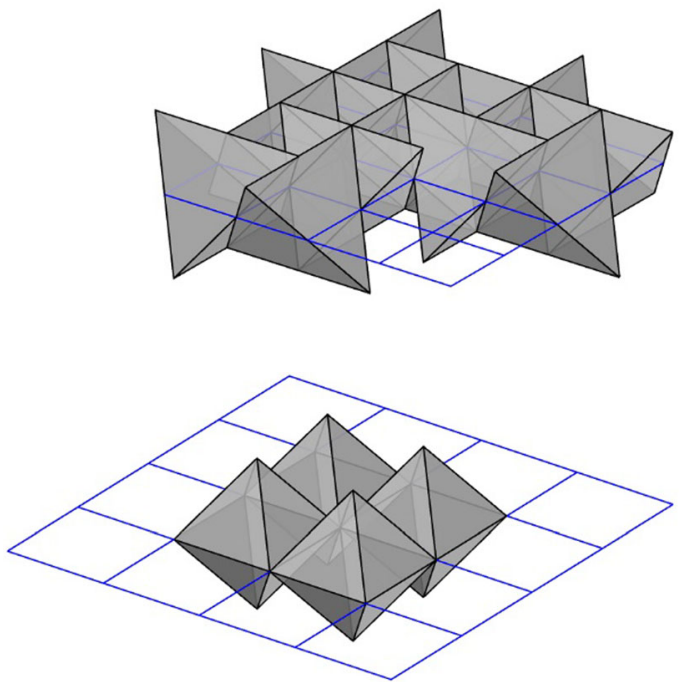

Fig. 35 Dodecahedra can be assembled with square facets outlining $\left(4^{4}\right)$, but they would not hold as an interlocked assembly (These dodecahedra and endo-dodecahedra filling the gaps between them fill space (Conway et al. 2008: 328))

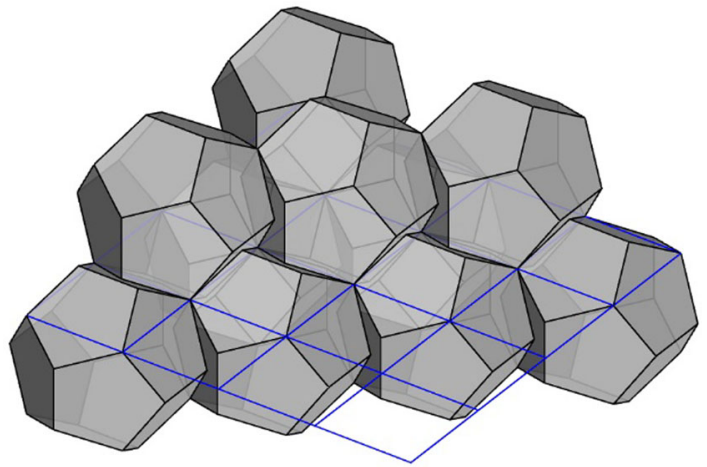


Fig. 36 Cuboctahedra can be assembled with square facets outlining $\left(4^{4}\right)$, but each pair has one coincident face

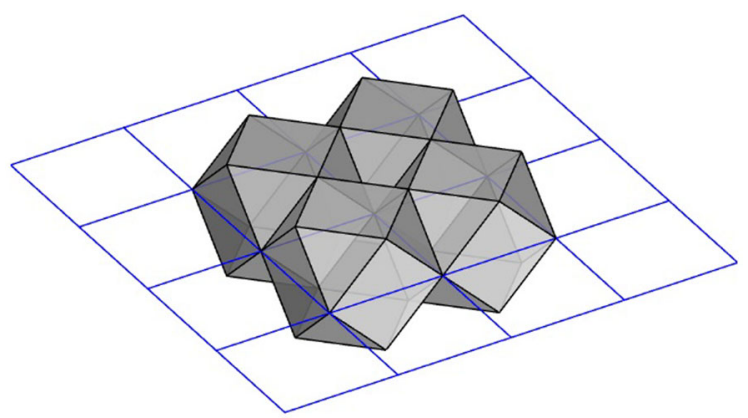

Fig. 37 It is not possible to assemble more than two icosidodecahedra with square facets according to $\left(4^{4}\right)$, without occurring intersections

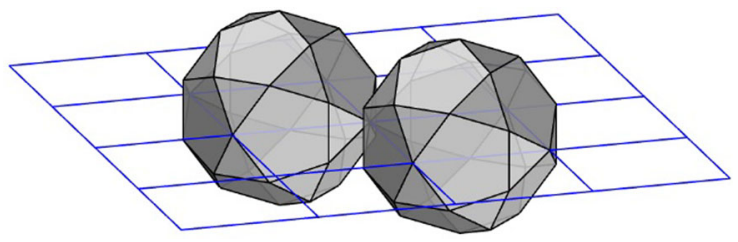

Fig. 38 Topological interlocking of cubes with hexagonal cross-sections outlining $\left(6^{3}\right)$

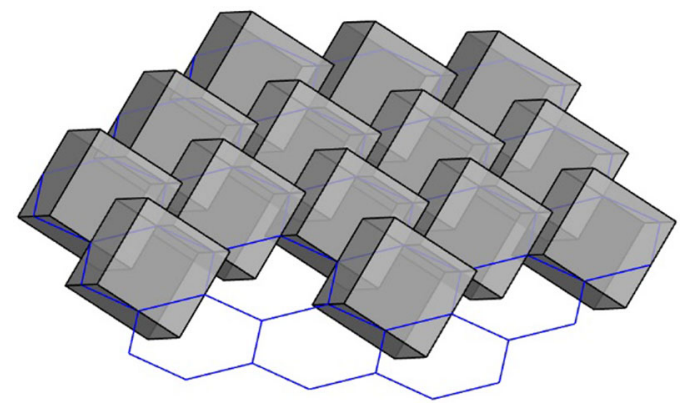

Fig. 39 Topological

interlocking of octahedra with hexagonal cross-sections outlining $\left(6^{3}\right)$

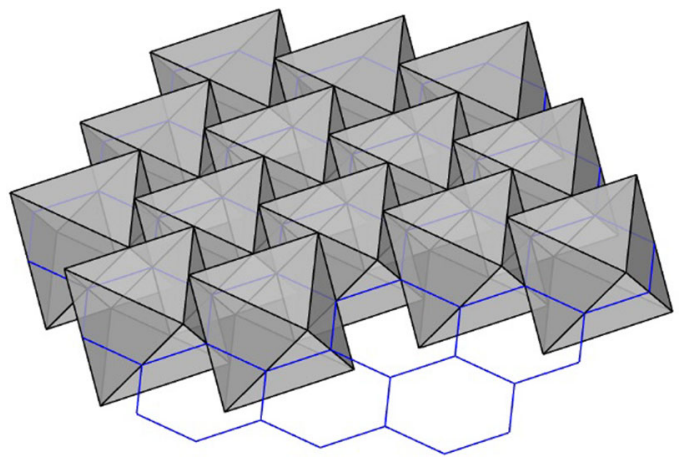

expands the number of interlocking assemblies with platonic solids referred by Kanel-Belov et al. (2008: 7). Icosidodecahedra (Fig. 32) can be assembled as an interlocking system as well, although these are structurally more complex polyhedra 
Fig. 40 Topological

interlocking of dodecahedra with hexagonal cross-sections outlining $\left(6^{3}\right)$
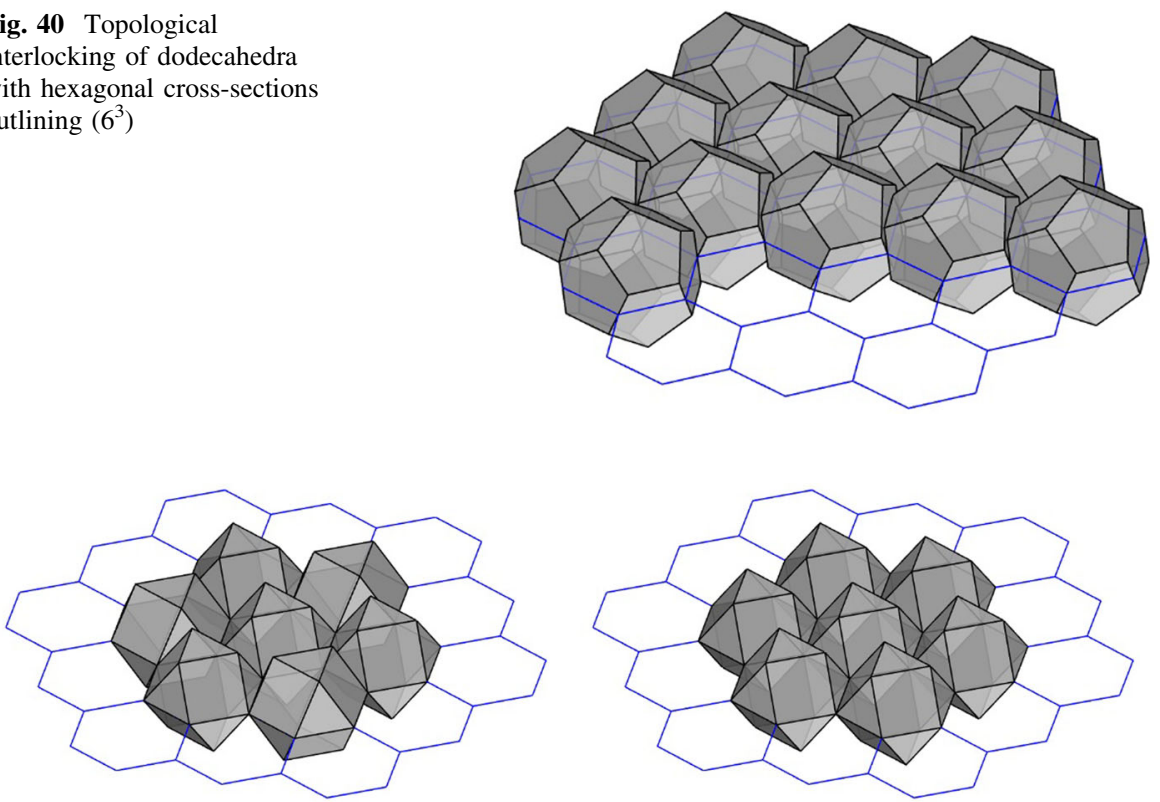

Fig. 41 Cuboctahedra can be assembled with hexagonal facets outlining $\left(6^{3}\right)$, but coplanar faces have only one edge in common
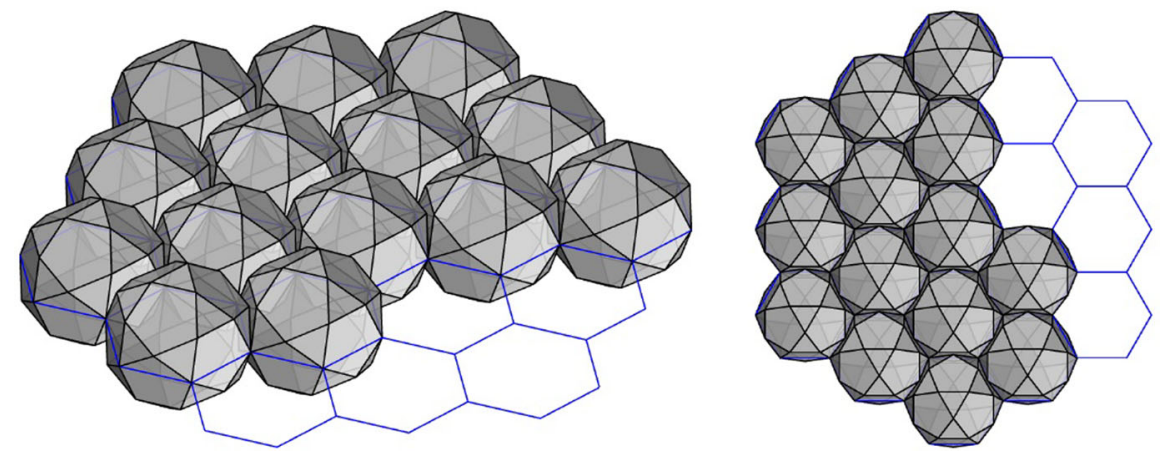

Fig. 42 Topological interlocking of icosidodecahedra with hexagonal facets outlining $\left(6^{3}\right)$, corresponding to the situation depicted in Fig. 32

than the octahedra in Fig. 39, whose section also degenerates into an equilateral triangle. But regarding plausible rejections for convex quasiregular polyhedra as elements of topological interlocked systems because of their inner complexity, if further investigations conclude these to be structurally efficient and somehow advantageous for stereotomic architecture, it is worth recalling what Weizmann et al. (2016) mention: "recent technological developments in digital design and fabrication seem to dissolve the limits in designing and fabricating complex architectural forms" (2016: 18). They also add that the geometrical complexity of 
Fig. 43 Dodecahedra with coplanar decagonal crosssections

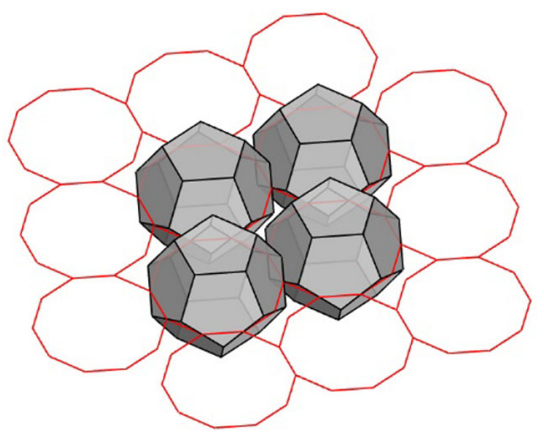

Fig. 44 Icosahedra with coplanar decagonal crosssections

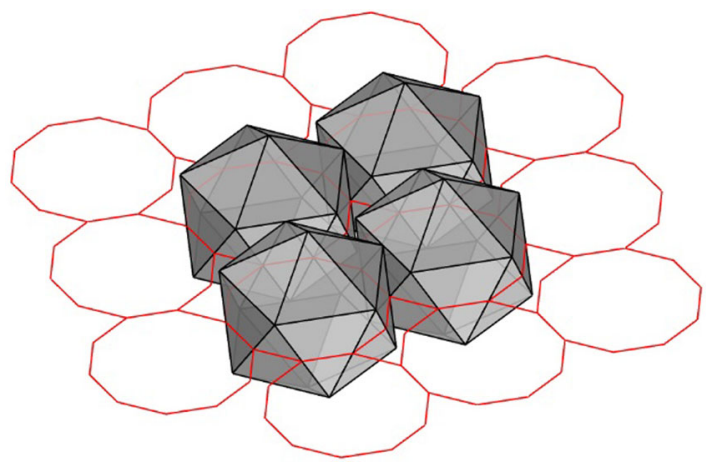

Fig. 45 Topological interlocking of dodecahedra with coplanar decagonal crosssections

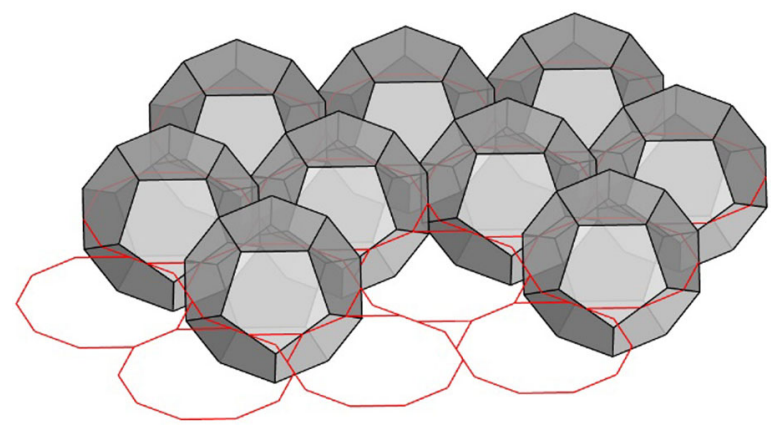

blocks should not be considered a limitation in itself, since "computer design and fabrication software tools and machines... can handle complex geometry" (2016: 22). A remark to denote that the assemblies in Figs. 32 and 42 are the same, because the triangular and hexagonal facets of the icosidodecahedra have common vertices.

Figures 33, 34, 35, 36 and 37 illustrate the possibilities that can be outlined from the tessellation $\left(4^{4}\right)$, beginning with the well-known interlocking of regular tetrahedra conceived by Glickman (1984) and later developed in Dyskin et al. (2001) who demonstrated that tetrahedron-shaped blocks in positions similar to Fig. 33 hold in place by their neighbouring blocks and that, together, they outline a 
Fig. 46 Topological interlocking of icosahedra with coplanar decagonal crosssections

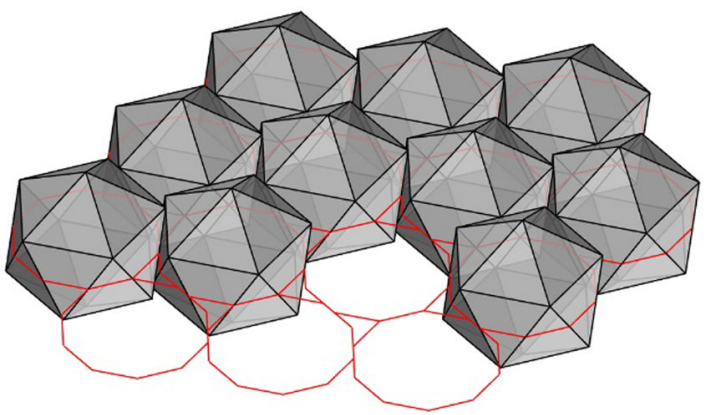

layer that is "flexible, but can withstand considerable loads even if no binder is used to hold the elements together" (2001: 2694). In conclusion, regular tetrahedra (and, by extension, every isosceles tetrahedron) can effectively be assembled in interlocked structures according to $\left(4^{4}\right)$, since the cross-section containing the edges' midpoints perpendicular to any twofold symmetry axis is a square. In this regard, it is important to highlight that the sum of adjacent dihedral angles between the tessellation's plane and each pair of coplanar faces $\left(54.74^{\circ}\right.$ and $125.26^{\circ}$ respectively) equals $180^{\circ}$.

Tessellation $\left(6^{3}\right)$ is probably the most prolific, but not every situation holds as a candidate for an interlocked assembly, as Fig. 41 demonstrates. Given that the dihedral angles between coplanar faces and the tessellation plane are supplementary, cubes (Fig. 38), octahedra (Fig. 39) and dodecahedra (Fig. 40) with coplanar hexagonal cross-sections outlining $\left(6^{3}\right)$ topologically interlock, as demonstrated in Dyskin et al. (2003: 3). Figure 41 shown examples of the infinite arrangements in which cuboctahedra might be assembled with their equatorial hexagons outlining $\left(6^{3}\right)$. However, none of their coplanar faces have more than one edge in common. Figure 42 is a geometrical possibility for a topological interlocked assembly, given that, corresponding to the exact situation depicted in Fig. 32, one of the threefold axis of each icosidodecahedron is perpendicular to the tessellation plane and the cross-section degenerates into an equilateral triangle.

Figures 43 and 44 illustrate the possibilities of assembling dodecahedra or icosahedra with coplanar decagonal cross-sections, that here fail to outline uniform tessellations and interlocked assemblies, since the gap between each set of four is a concave equilateral hexagon. Regular decagons (as regular pentagons) cannot tessellate the (Euclidean) plane because of their internal angles $\left(144^{\circ}\right.$ and $108^{\circ}$ respectively). Correspondingly, convex polyhedra of the icosahedral symmetry group ${ }^{8}$ are not found in uniform solid tessellations. In each case, coplanar faces of polyhedra intersect in intercalate sides of the decagons, and pairs of polyhedra share a common rhombus, whose minor diagonal is one of the decagon's sides.

\footnotetext{
8 The following convex uniform polyhedra belong to the icosahedral group and have thirty-one axes of rotational symmetry (fifteen 2 -fold, ten 3 -fold and six 5-fold axes): the dodecahedron, the icosahedron, the icosidodecahedron, the rhombicosidodecahedron, the snub dodecahedron, the truncated dodecahedron and the truncated icosahedron.
} 
Figures 45 and 46 illustrate the topological interlocking of dodecahedra and icosahedra presented in Kanel-Belov et al. (2008: 12), in which the planar gaps between the cross-sections have the least possible area, coplanar faces of polyhedra intersect in every decagons' side, each pair of dodecahedra and icosahedra have more than small rhombuses in common and every triad of polyhedra interlock.

\section{Conclusion}

We have analysed all uniform tessellations in three-dimensional space, in which convex polyhedra meet face-to-face to fill space in vertex-transitive arrangements and explored the relations between them and its underlying uniform plane tessellations. Additionally, we have illustrated the Architectonic tessellations and the corresponding catoptric chamber that, through mirror-reflections, outline an interesting set of monohedral tessellations. The interrelations between solid and plane tessellations were intended to clarify the subject of topological interlocking, to which we proposed a systematic approach and two new geometrical possibilities for interlocked assemblies. Further research will evaluate the effectiveness of our systematic study and identify which structural predicaments occur in the proposed assemblies and, if similar to the "negative stiffness discovered in the interlocking assemblies of cubes" observed in Estrin et al. (2011: 1192), its relations to the external constraint configuration. Our next investigation will focus on a systematic analysis of the geometrical possibilities for topological interlocked assemblies with convex semiregular polyhedra in the eleven uniform plane tessellations.

Acknowledgements All images are from the author, except for Fig. 7, retrieved from http://media. accademiaxl.it/memorie/S3-VXIV-1907/Andreini75-129.pdf in October 2017 and in the public domain. 


\section{Appendix ${ }^{9}$}

APPENDIX - CONVEX UNIFORM TESSELLATIONS IN EUCLIDEAN SPACE (1/4)

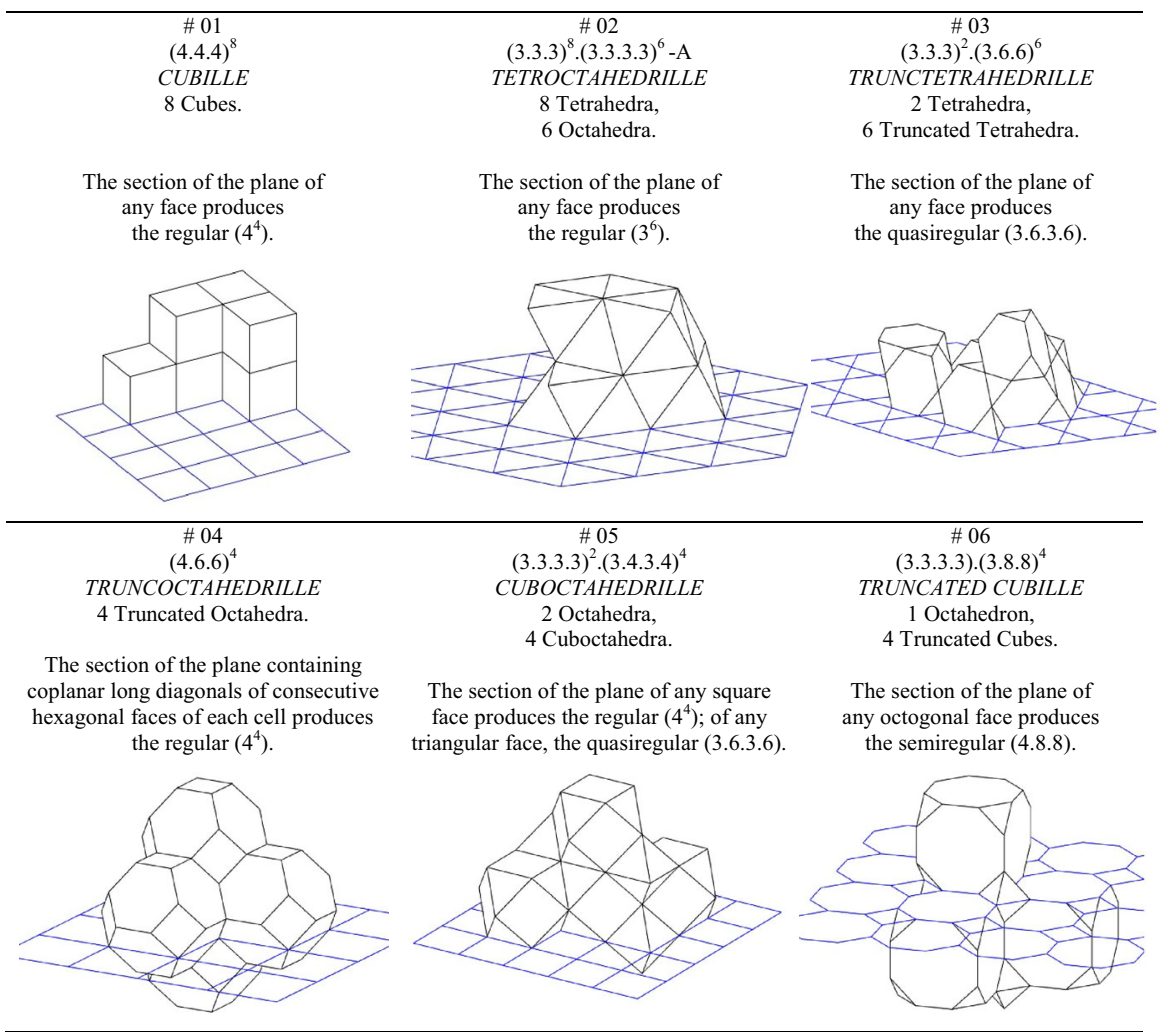

\footnotetext{
9 Descriptions of each solid tessellation were adapted from (Pearce 1978: 41-50), (Grunbaum 1994:
} 54-56), (Conway et al. 2008: 292-298) and (Wikipedia contributors 2018). 
APPENDIX - CONVEX UNIFORM TESSELLATIONS IN EUCLIDEAN SPACE (2/4)

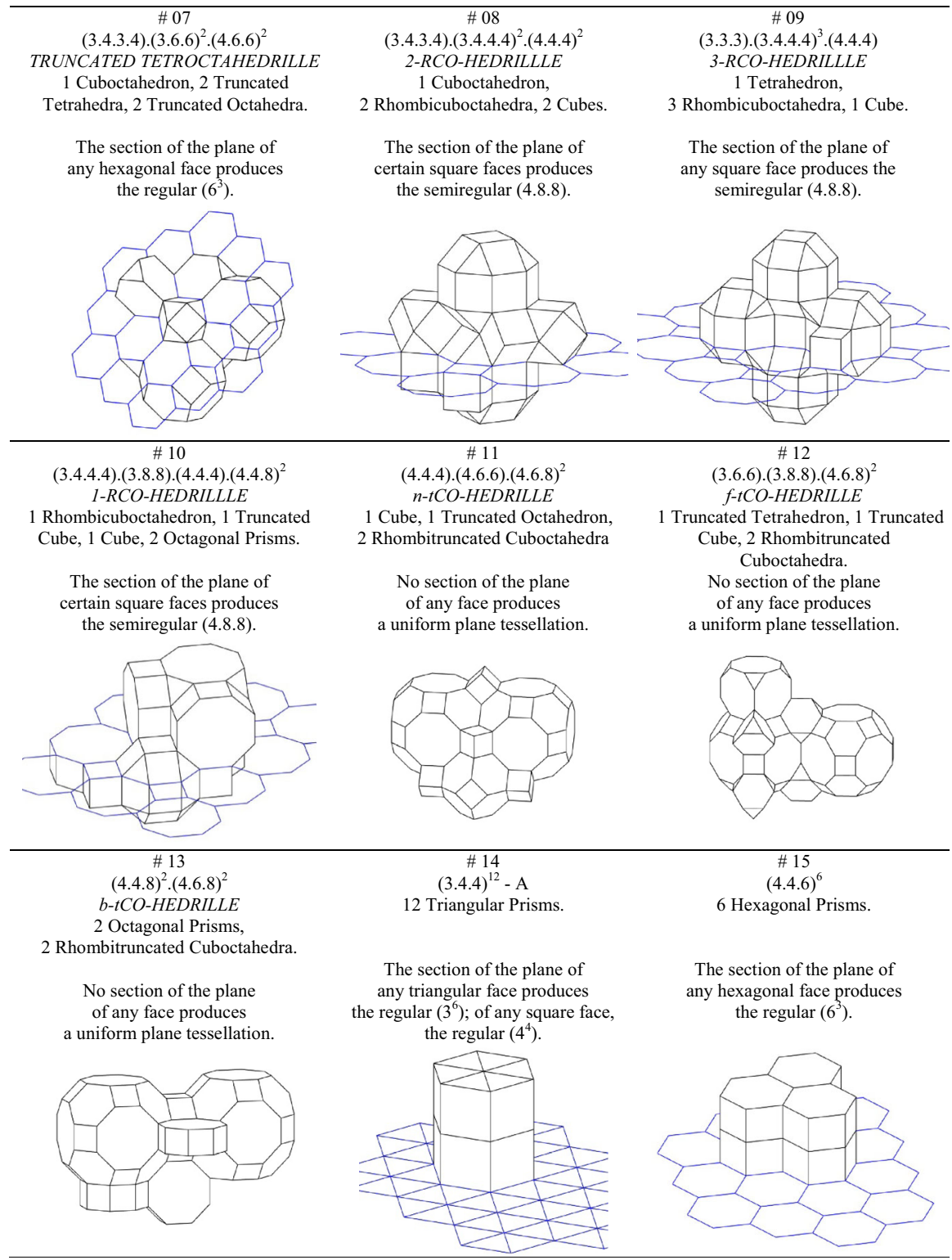




\section{APPENDIX - CONVEX UNIFORM TESSELLATIONS IN EUCLIDEAN SPACE (3/4)}

\section{\# 16}

$(3.4 .4)^{8} .(4.4 .6)^{2}$

8 Triangular prisms,

2 Hexagonal Prisms

The section of the plane of any triangular or hexagonal face produces the semiregular (3.3.3.3.6), of which 2 enatiomorphics versions exist.

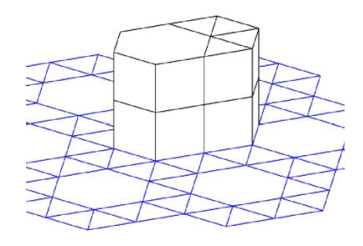

\# 19

$(3.4 .4)^{4} \cdot(4.4 \cdot 6)^{4}$

4 Triangular Prisms,

4 Hexagonal Prisms.

The section of the plane of any triangular or hexagonal face produces the quasiregular (3.6.3.6).

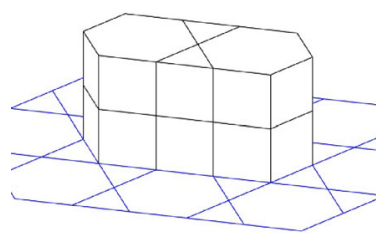

$\# 22$

$(4.4 .4)^{2} \cdot(4.4 \cdot 6)^{2} \cdot(4 \cdot 4 \cdot 12)^{2}$

2 Cubes, 2 Hexagonal Prisms, 2

Dodecagonal Prisms.

The section of the plane of any triangular or dodecagonal face (and certain square faces) produces the semiregular (4.6.12).

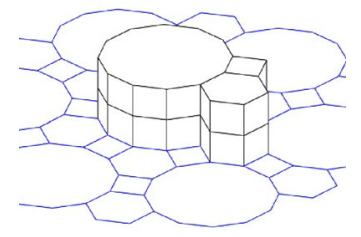

\section{$(3.4 .4)^{6} .(4.4 .4)^{4}-\mathrm{A}$ \\ 6 Triangular Prisms, 4 Cubes.}

The section of the plane of any triangular face produces the semiregular (3.3.3.4.4); of certain square faces, the regular $\left(4^{4}\right)$.

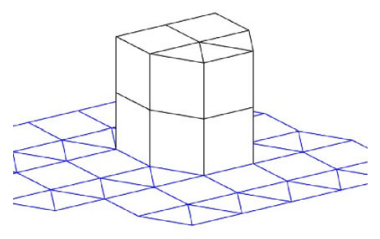

$$
\text { \#20 }
$$

$(3.4 .4)^{2} \cdot(4.4 .4)^{4} \cdot(4.4 .6)^{2}$

2 Triangular Prisms,

4 Cubes, 2 Hexagonal Prisms.

The section of the plane of any triangular or hexagonal face (and certain square faces) produces the semiregular (3.4.6.4).

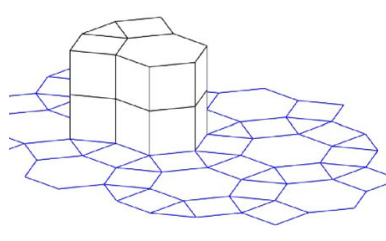

\# 23

$(4.4 .4)^{2} \cdot(4.4 .8)^{4}$ 2 Cubes,

4 Octagonal Prisms.

The section of the plane of any octagonal face (and certain

square faces) produces the semiregular (4.8.8).

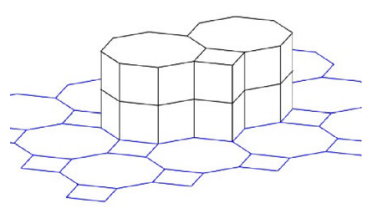

\section{\# 18}

$(3.4 .4)^{6} \cdot(4.4 .4)^{4}-\mathrm{B}$

6 Triangular Prisms, 4 Cubes.

The section of the plane of any triangular face (and certain square faces) produces the semiregular (3.3.4.3.4).

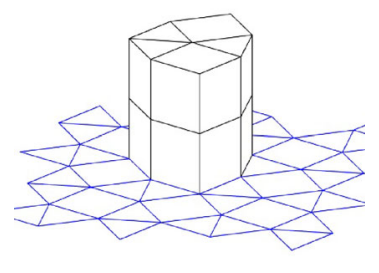

\section{\# 21}

$(3.4 .4)^{2} .(4.4 .12)^{4}$

2 Triangular Prisms,

4 Dodecagonal Prisms.

The section of the plane of any triangular or dodecagonal

face produces the

semiregular (3.12.12).

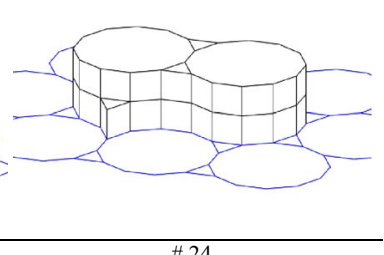

$(3.3 \cdot 3)^{8} \cdot(3.3 \cdot 3 \cdot 3)^{6}-\mathrm{B}$

8 Tetrahedra, 6 Octahedra.

Gyrated version of

$(3.3 \cdot 3)^{8} \cdot(3 \cdot 3 \cdot 3 \cdot 3)^{6}-\mathrm{A}$.

The section of the plane of certain faces produces the regular $\left(3^{6}\right)$.

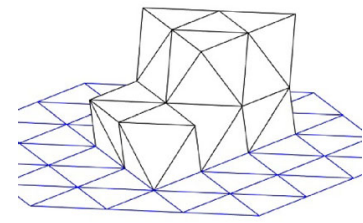




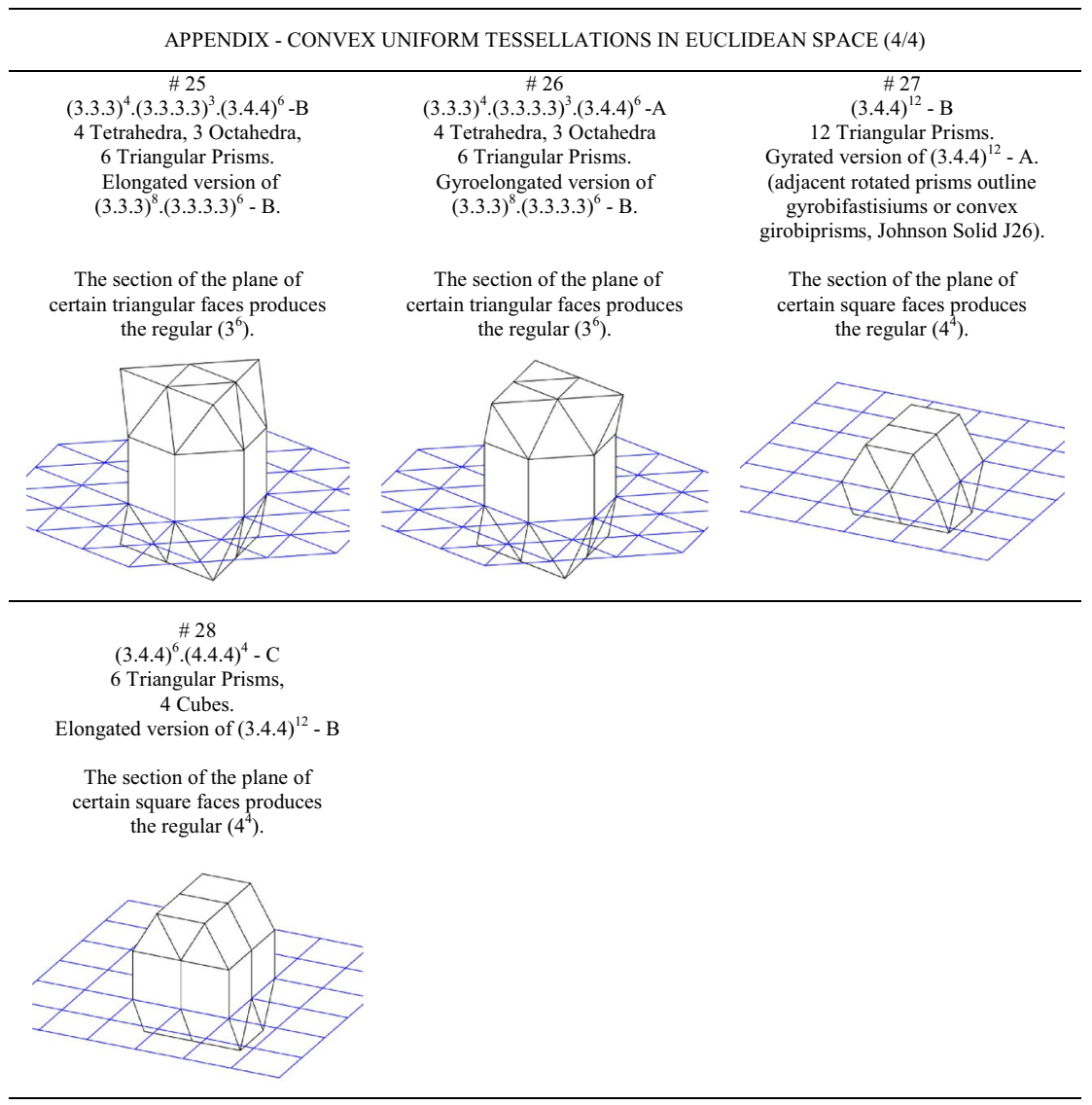

\section{References}

Andreini, A. 1905. Sulle reti di poliedri regolari e semiregolari e sulle corrispondenti reti correlative. Mem. Società Italiana della Scienze, Ser. 3, 14: 75-129. Retrieved from http://media.accademiaxl.it/ memorie/S3-VXIV-1907/Andreini75-129.pdf in October 2017.

Ball, W., Coxeter, H. \& Ball, W. 1987. Mathematical Recreations and Essays. New York: Dover Publications.

Chieh, C., 1988. Polyhedra and Crystal Structures. In Shaping Space: A Polyhedral Approach, M. Senechal, M. \& G. M. Fleck, G. M., eds., 118-132. Boston: Birkhäuser.

Conway, J., Burgiel, H. \& Goodman-Strauss, C. 2008. The symmetries of things. Wellesley, MA: A.K. Peters.

Coxeter, H. 1973. Regular Polytopes. New York: Dover Publications.

Critchlow, K. 1969. Order in Space: a design source book. London: Thames and Hudson.

Cromwell, P. 1997. Polyhedra. Cambridge, U.K.: Cambridge University Press.

Deza, M. \& Shtogrin, M. 2000. Uniform Partitions of 3-space, their Relatives and Embedding. European Journal of Combinatorics, 21(6), 807-814. https://doi.org/10.1006/eujc.1999.0385.

Dyskin, A., Estrin, Y., Kanel-Belov, A. \& Pasternak, E. 2003. Topological interlocking of platonic solids: A way to new materials and structures. Philosophical Magazine Letters, 83(3): 197-203. https://doi. org/10.1080/0950083031000065226. 
Dyskin, A., Estrin, Y., Kanel-Belov, A. \& Pasternak, E. 2001. A new concept in design of materials and structures: Assemblies of interlocked tetrahedron-shaped elements. Scripta Materialia, 44(12), 2689-2694. https://doi.org/10.1016/s1359-6462(01)00968-x.

Estrin, Y., Dyskin, A., \& Pasternak, E. 2011. Topological interlocking as a material design concept. Materials Science and Engineering: C, 31(6): 1189-1194. https://doi.org/10.1016/j.msec.2010.11. 011.

Gheorghiu, A. \& Dragomir, V. 1978. Geometry of structural forms. London: Applied Science Pub.

Glickman. M., 1984. The G-Block System of Vertically Interlocking Paving. Proceedings of the 2nd International Conference on Concrete Block Paving, 10-12. Delft.

Grünbaum, B. 2010. The Bilinski Dodecahedron and Assorted Parallelohedra, Zonohedra, Monohedra, Isozonohedra, and Otherhedra. The Mathematical Intelligencer 32(4): 5-15. https://doi.org/10.1007/ s00283-010-9138-7.

Grünbaum, B. 1994. Uniform tilings of 3-space. Geombinatorics 4: 49-56.

Grünbaum, B. \& Shepard, G. 1987. Tilings and Patterns. New-York: W. H. Freeman \& Company.

Grünbaum, B. \& Shephard, G. C. 1980. Tilings with congruent tiles. Bulletin of the American Mathematical Society 3(3): 951-974. https://doi.org/10.1090/s0273-0979-1980-14827-2.

Holden, A. 1971. Shapes, space, and symmetry. New York: Columbia University Press.

Kanel-Belov A., Dyskin, A., Estrin, Y., Pasternak, E. \& Ivanov-Pogodaev, I. 2008. Interlocking of Convex Polyhedra: Towards a Geometric Theory of Fragmented Solids (ArXiv08125089 Math).

Kappraff, J. 1990. Connections: The geometric bridge between art and science. New York: McGraw-Hill. Lalvani, H. 1992. Continuous Transformations of Non-Periodic Tilings and Space-Fillings. Fivefold Symmetry, 97-128. https://doi.org/10.1142/9789814439497_0006.

Malkevitch, J. 1988. Milestones in the History of Polyhedra. In Shaping Space: A Polyhedral Approach, M. Senechal, M. \& G. M. Fleck, G. M., eds., 80-92. Boston: Birkhäuser.

Olshevsky, G. 2006. Uniform Panoploid Tetracombs, Manuscript. Retrieved from http://ajo. tabletoptelephone.com/ hopspage/Fuller.html in April 2016.

Pugh, A. 1976. Polyhedra: A Visual Approach. Berkeley: University of California Press.

Pearce, P. 1978. Structure In Nature Is A Strategy For Design. Cambridge: MIT Press.

Steinhaus, H. 1960. Mathematical Snapshots. $2^{\text {nd }}$ ed. New York: Oxford University Press.

Towle, R. 1996. Polar Zonohedra, The Mathematica Journal, 1996 Retrieved April 26, 2018 http://www. mathematica-journal.com/issue/v6i2/graphics/towle.pdf.

Weizmann, M., Amir, O. \& Grobman, Y. 2016. Topological interlocking in buildings: A case for the design and construction of floors. Automation in Construction, 72: 18-25. https://doi.org/10.1016/j. autcon.2016.05.014.

Wenninger, M. 1971. Polyhedron models. Cambridge: University Press.

Wikipedia contributors. 2018, January 26. Convex uniform honeycomb. In Wikipedia, The Free Encyclopedia. Retrieved April 7, 2018 from https://en.wikipedia.org/w/index.php?title=Convex_ uniform_honeycomb\&oldid=822420094.

Williams, R. 1979. The Geometrical Foundation of Natural Structure: A Source Book of Design. New York: Dover Publications.

Vera Viana graduated in Painting in the Fine Arts Faculty of the University of Porto, Portugal. As an integrated Researcher at the CEAU/FAUP (Faculty of Architecture of Porto's University), her research focuses on polyhedral geometry and the relationships between architecture and mathematics. Presently taking a Ph.D. focusing on polyhedra and spatial tessellations at the Sciences and Technology School (Didactic of Mathematical Sciences) in UTAD (Trás-os-Montes and Alto Douro's University). Director of Aproged (Portuguese Geometry and Drawing Teachers' Association) since 2009, she organizes the International Conferences Geometrias (since 2013), Jornadas Didácticas (since 2016), Aproged's National Meetings (2008-2012) and is Editor-in-Chief of the Geometrias Conference Proceedings and Aproged's Bulletins (since 2009). She has been the Conference Report Editor for the Nexus Network Journal since 2014. As a teacher of descriptive geometry, a teacher trainer (on the exploration of dynamic geometry software), authorized Rhino Trainer and author of two schoolbooks on descriptive geometry for the secondary level (2013 and 2014), Viana has been engaged since 2001 in the development of educational resources with dynamic geometry, three-dimensional modelling and algorithmic modelling software, having authored papers and presentations on the subject. 